\title{
A CALCULUS FOR THE MODULI SPACE OF CURVES
}

\author{
R. PANDHARIPANDE
}

\section{INTRODUCTION}

The moduli space $\mathcal{M}_{g}$ of complete nonsingular curves of genus $g$ admits a compactification

$$
\mathcal{M}_{g} \subset \overline{\mathcal{M}}_{g}
$$

by stable curves. Mumford, in Towards an enumerative geometry of the moduli space of curves (published in 1983), writes:

The goal of this paper is to formulate and to begin an exploration of the enumerative geometry of the set of all curves of arbitrary genus $g$. By this we mean setting up a Chow ring for the moduli space $\mathcal{M}_{g}$ and its compactification $\overline{\mathcal{M}}_{g}$, defining what seem to be the most important classes in this ring and calculating the class of some geometrically important loci in $\overline{\mathcal{M}}_{g}$ in terms of these classes. We take as a model for this the enumerative geometry of the Grassmannians. [65]

Mumford's most important classes are now termed tautological classes. He opened the door to the study of their algebra - a fascinating topic connected to many areas of modern mathematics.

More than three decades have passed since Mumford's article. The progress in our understanding of the intersection theory of the moduli space of curves has been considerable. Calculations by classical methods of the algebra of tautological classes on $\mathcal{M}_{g}$ for low $g$ by Faber [18], starting in the 80's and continuing later in the 90's with Zagier, have proved to be fundamental. Witten's conjecture [88] in the 90's relating the integration of the cotangent line classes on the moduli spaces $\overline{\mathcal{M}}_{g, n}$ of stable pointed curves to the KdV hierarchy was a marvelous

Date: April 2016. 
surprise: the study of the algebra of tautological classes was linked at a basic level to the theory of integrable hierarchies. The deep role of topology was highlighted in 2007 by the landmark proof of Madsen and Weiss [59] via homotopy theory of Mumford's conjecture on the stable cohomology of $\mathcal{M}_{g}$ as $g \rightarrow \infty$.

Starting in the mid 90's, there was a swift development of GromovWitten theory. The moduli space $\overline{\mathcal{M}}_{g, n}(X)$ of stable maps intertwines the geometry of $\overline{\mathcal{M}}_{g, n}$ with the geometry of the nonsingular target variety $X$. Gromov-Witten theory is based upon the virtual fundamental class [2, 3] of the moduli of stable maps,

$$
\left[\overline{\mathcal{M}}_{g, n}(X)\right]^{v i r} \in A_{*}\left(\overline{\mathcal{M}}_{g, n}(X)\right)
$$

a new algebraic cycle ${ }^{1}$ whose properties constrain the algebra of tautological classes of $\overline{\mathcal{M}}_{g, n}$ in remarkable ways.

A systematic study of the constraints imposed by Gromov-Witten theory on the algebra of tautological classes was started 2009 in [69] and continued in [70, 71]. Recent progress has culminated in a complete proposal by Pixton [79] for a calculus of tautological classes on $\overline{\mathcal{M}}_{g, n}$.

My goal here is to present Pixton's proposal and survey the rapid advances of the past 6 years. Several open questions are discussed. An effort has been made to condense a great deal of mathematics into as few pages as possible with the hope that the reader will follow through to the end.

Acknowledgments. The spirit of my lecture at the 2015 AMS summer institute in algebraic geometry in Salt Lake City has been followed rather closely here. I would like to thank the Clay Mathematics Institute for supporting my visit. Some of the material in Sections 1-3 is based on unpublished notes [68] of a lecture I gave at $A$ celebration of algebraic geometry at Harvard in 2011. Sections 4 and 5 are directly connected to the lecture in Salt Lake City. A discussion of the recent

\footnotetext{
${ }^{1}$ All Chow (and cohomology) groups in the paper will be taken with $\mathbb{Q}$ coefficients.

${ }^{2}$ I have dated Theorems 1-7 presented in the paper (and the surrounding results) by the years in which the proofs were found. Published versions appear later and in mixed order. The dates of publication can be found in the bibliography.
} 
formula [61] for the Chern characters of the Verlinde bundle on $\overline{\mathcal{M}}_{g, n}$ was presented in the Salt Lake City lecture, but is omitted here.

Much of what I know about the moduli space of curves has been learned through collaborations. Directly relevant to the material presented here is work with P. Belorousski, C. Faber, G. Farkas, E. Getzler, T. Graber, F. Janda, X. Liu, A. Marian, A. Okounkov, D. Oprea, A. Pixton, and D. Zvonkine. Discussions in Zürich with A. Buryak, R. Cavalieri, E. Clader, D. Petersen, O. Randal-Williams, Y. Ruan, I. Setayesh, and Q. Yin have played an important role. I have been very fortunate to have had the opportunity to interact with all of these mathematicians.

I am supported by the grants SNF-200020162928 and ERC-2012AdG-320368-MCSK, and SwissMAP. I am also supported by the Einstein Stiftung in Berlin.

\section{Tautological Classes on $\mathcal{M}_{g}$}

1.1. $\kappa$ classes. Let $\mathcal{M}_{g}$ be the moduli space of complete nonsingular genus $g \geq 2$ curves over $\mathbb{C}$, and let

$$
\pi: \mathcal{C}_{g} \rightarrow \mathcal{M}_{g}
$$

be the universal curve. We view $\mathcal{M}_{g}$ and $\mathcal{C}_{g}$ as nonsingular, quasiprojective, Deligne-Mumford stacks. However, the orbifold perspective is sufficient for most of our purposes.

The cotangent line $\mathbb{L}$ to the fibers of the morphism (1) defines a cotangent line class,

$$
\psi=c_{1}(\mathbb{L}) \in A^{1}\left(\mathcal{C}_{g}\right) .
$$

The $\kappa$ classes are defined by push-forward,

$$
\kappa_{r}=\pi_{*}\left(\psi^{r+1}\right) \in A^{r}\left(\mathcal{M}_{g}\right) .
$$

The tautological ring

$$
R^{*}\left(\mathcal{M}_{g}\right) \subset A^{*}\left(\mathcal{M}_{g}\right)
$$

is the $\mathbb{Q}$-subalgebra generated by all of the $\kappa$ classes.$^{3}$ There is a canonical quotient

$$
\mathbb{Q}\left[\kappa_{1}, \kappa_{2}, \kappa_{3}, \ldots\right] \stackrel{q}{\longrightarrow} R^{*}\left(\mathcal{M}_{g}\right) \longrightarrow 0 \text {. }
$$

\footnotetext{
${ }^{3}$ Since $\kappa_{0}=2 g-2 \in \mathbb{Q}$ is a multiple of the fundamental class, we need not take $\kappa_{0}$ as a generator.
} 
The kernel of $q$ is the ideal of relations among the $\kappa$ classes.

1.2. Motivations. There are two basic motivations for the study of the tautological rings $R^{*}\left(\mathcal{M}_{g}\right)$. The first is Mumford's conjecture proven by Madsen and Weiss [59],

$$
\lim _{g \rightarrow \infty} H^{*}\left(\mathcal{M}_{g}\right)=\mathbb{Q}\left[\kappa_{1}, \kappa_{2}, \kappa_{3}, \ldots\right]
$$

determining the stable cohomology of the moduli of curves. While the $\kappa$ classes do not exhaust $H^{*}\left(\mathcal{M}_{g}\right)$, there are no other stable classes.

The second motivation comes from a large body of classical calculations on $\mathcal{M}_{g}$ (often related to Brill-Noether theory). The answers invariably lie in the tautological ring $R^{*}\left(\mathcal{M}_{g}\right)$. The study of tautological classes by Mumford [65] was directly inspired by such algebro-geometric cycle constructions.

1.3. Schubert calculus. The structure of the Chow ring of the Grassmannian $\operatorname{Gr}(r, n)$ of $r$-dimensional subspaces of $\mathbb{C}^{n}$ is well-known [26] and may be viewed as a model for the study of the tautological classes on $\mathcal{M}_{g}$.

The Chern classes of the universal subbundle

$$
\mathrm{S} \rightarrow \mathrm{Gr}(r, n)
$$

generate the entire Chow ring,

$$
\mathbb{Q}\left[c_{1}(\mathrm{~S}), \ldots, c_{r}(\mathrm{~S})\right] \stackrel{q}{\longrightarrow} A^{*}(\mathrm{Gr}(r, n)) \longrightarrow 0 .
$$

The kernel of $q$ is expressed in term of the Segre classes of the universal subbundle as

$$
\operatorname{ker}(q)=\left(s_{n-r+1}(\mathrm{~S}), \ldots, s_{n}(\mathrm{~S})\right), \quad \frac{1}{c(\mathrm{~S})}=s(\mathrm{~S}) .
$$

The Schubert calculus for the Grassmannian yields classical formulas for geometric loci in terms of the generators $c_{i}(\mathrm{~S})$. The subject is fundamentally connected to the representation theory of the symmetric group.

A basic goal (expressed in the quotation of Mumford in the Introduction) is to develop a calculus for tautological classes on the moduli space of curves parallel to the Schubert calculus for the Grassmannian. 
1.4. Cohomology. We may also define a tautological ring

$$
R H^{*}\left(\mathcal{M}_{g}\right) \subset H^{*}\left(\mathcal{M}_{g}\right)
$$

generated by the $\kappa$ classes in cohomology. Since there is a natural factoring

$$
\mathbb{Q}\left[\kappa_{1}, \kappa_{2}, \kappa_{3}, \ldots\right] \stackrel{q}{\longrightarrow} R^{*}\left(\mathcal{M}_{g}\right) \stackrel{c}{\longrightarrow} R H^{*}\left(\mathcal{M}_{g}\right)
$$

via the cycle class map $c$, algebraic relations among the $\kappa$ classes are also cohomological relations. Whether or not there exist more cohomological relations is not yet settled.

Q1. Is the cycle class map $R^{*}\left(\mathcal{M}_{g}\right) \stackrel{c}{\longrightarrow} R H^{*}\left(\mathcal{M}_{g}\right)$ an isomorphism?

Calculations (discussed in Section 2.3 below) show the answer to question Q1 is affirmative at least for $g<24$.

\section{FABER-Zagier RElations on $\mathcal{M}_{g}$}

2.1. Conjecture and proof. Guided by low genus calculations and deep insight, Faber and Zagier conjectured in 2000 a remarkable set of relations among the $\kappa$ classes in $R^{*}\left(\mathcal{M}_{g}\right)$ for all $g$.

The first proof [70, 71] of the Faber-Zagier conjecture (Theorem 1] of Section 2.2 was given in 2010 via a geometric construction involving the virtual class of the moduli space of stable quotients [60] S Since then, a proof [72] in $R H^{*}\left(\mathcal{M}_{g}\right)$ via Witten's 3-spin class was found in 2013, and a second proof [40, 42] in $R^{*}\left(\mathcal{M}_{g}\right)$ via the virtual class of the moduli space of stable maps to $\mathbb{P}^{1}$ was found in 2014.

2.2. Formulas. To write the Faber-Zagier relations, we will require the following notation. Let the variable set

$$
\mathbf{p}=\left\{p_{1}, p_{3}, p_{4}, p_{6}, p_{7}, p_{9}, p_{10}, \ldots\right\}
$$

\footnotetext{
${ }^{4}$ The first relations obtained by virtual localization 32 on the moduli space of stable quotients can be found in [60, Section 8]. See also 89] for a study of properties of the relations of [60, Section 8].
} 
be indexed by positive integers not congruent to 2 modulo 3 . Define the series

$$
\begin{aligned}
\Psi(t, \mathbf{p})=\left(1+t p_{3}\right. & \left.+t^{2} p_{6}+t^{3} p_{9}+\ldots\right) \sum_{i=0}^{\infty} \frac{(6 i) !}{(3 i) !(2 i) !} t^{i} \\
& +\left(p_{1}+t p_{4}+t^{2} p_{7}+\ldots\right) \sum_{i=0}^{\infty} \frac{(6 i) !}{(3 i) !(2 i) !} \frac{6 i+1}{6 i-1} t^{i} .
\end{aligned}
$$

Since $\Psi$ has constant term 1, we may take the logarithm. Define the constants $C_{r}^{\mathrm{FZ}}(\sigma)$ by the formula

$$
\log (\Psi)=\sum_{\sigma} \sum_{r=0}^{\infty} C_{r}^{\mathrm{FZ}}(\sigma) t^{r} \mathbf{p}^{\sigma} .
$$

The above sum is over all partitions ${ }^{5} \sigma$ of size $|\sigma|$ which avoid parts congruent to 2 modulo 3 . The empty partition is included in the sum. Following standard conventions, to the partition

$$
\sigma=1^{n_{1}} 3^{n_{3}} 4^{n_{4}} \ldots
$$

we associate the monomial $\mathbf{p}^{\sigma}=p_{1}^{n_{1}} p_{3}^{n_{3}} p_{4}^{n_{4}} \cdots$. Let

$$
\gamma^{\mathrm{FZ}}=\sum_{\sigma} \sum_{r=0}^{\infty} C_{r}^{\mathrm{FZ}}(\sigma) \kappa_{r} t^{r} \mathbf{p}^{\sigma} .
$$

For a series $\Theta \in \mathbb{Q}[\kappa][[t, \mathbf{p}]]$ in the variables $\kappa_{i}$, $t$, and $p_{j}$, let $[\Theta]_{t^{r} \mathbf{p}^{\sigma}}$ denote the coefficient of $t^{r} \mathbf{p}^{\sigma}$ (which is a polynomial in the $\kappa_{i}$ ).

Theorem 1 (P.-Pixton 2010). In $R^{d}\left(\mathcal{M}_{g}\right)$, the Faber-Zagier relation

$$
\left[\exp \left(-\gamma^{\mathrm{FZ}}\right)\right]_{t^{d} \mathbf{p}^{\sigma}}=0
$$

holds when $d>\frac{g-1+|\sigma|}{3}$ and $g \equiv d+|\sigma|+1 \bmod 2$.

The dependence upon the genus $g$ in the Faber-Zagier relations of Theorem 1 occurs in the inequality, the modulo 2 restriction, and via $\kappa_{0}=2 g-2$. For a given genus $g$ and codimension $r$, Theorem 1 provides only finitely many relations. While not immediately clear from the definition, the $\mathbb{Q}$-linear span of the Faber-Zagier relations determines an ideal in $\mathbb{Q}\left[\kappa_{1}, \kappa_{2}, \kappa_{3}, \ldots\right]$, see [71, Section 6$]$.

\footnotetext{
${ }^{5}$ All parts of a partition are positive (a condition satisfied by the empty partition).
} 
2.3. Gorenstein property. The ring $R^{*}\left(\mathcal{M}_{g}\right)$ is generated over $\mathbb{Q}$ by the classes

$$
\kappa_{1}, \kappa_{2}, \ldots, \kappa_{\left\lfloor\frac{g}{3}\right\rfloor} \in R^{*}\left(\mathcal{M}_{g}\right),
$$

as conjectured by Faber [18] and proven by Morita [64] in $R H^{*}\left(\mathcal{M}_{g}\right)$ and Ionel [38 in $R^{*}\left(\mathcal{M}_{g}\right)$. By Boldsen's results [7], there are no relations among the $\kappa$ classes of degree less than or equal to $\left\lfloor\frac{g}{3}\right\rfloor$. Looijenga's results [58] established following nonvanishing and vanishing conjectures of Faber [18]:

$$
R^{g-2}\left(\mathcal{M}_{g}\right) \cong \mathbb{Q}, \quad R^{>g-2}\left(\mathcal{M}_{g}\right)=0 .
$$

The proportionalities in $R^{g-2}\left(\mathcal{M}_{g}\right)$ of monomials in the $\kappa$ classes are known via Hodge integral evaluations [18, 19, 31]. The generation, nonvanishing, vanishing, and proportionality results were all conjectured by Faber in 90s and proven by 2005 .

For $g<24$, the Faber-Zagier relations yield ${ }^{6}$ a Gorenstein ring,

$$
R_{\mathrm{FZ}}^{*}\left(\mathcal{M}_{g}\right)=\frac{\mathbb{Q}\left[\kappa_{1}, \kappa_{2}, \kappa_{3}, \ldots\right]}{\text { FZ-relations }},
$$

with socle in codimension $g-2$. By the Gorenstein property, the pairing

$$
R_{\mathrm{FZ}}^{d}\left(\mathcal{M}_{g}\right) \times R_{\mathrm{FZ}}^{g-2-d}\left(\mathcal{M}_{g}\right) \rightarrow R_{\mathrm{FZ}}^{g-2}\left(\mathcal{M}_{g}\right) \cong \mathbb{Q}
$$

is nondegenerate for $0 \leq d \leq g-2$. The nondegeneracy of the pairing forbids additional relations, so

$$
R_{\mathrm{FZ}}^{*}\left(\mathcal{M}_{g}\right) \cong R^{*}\left(\mathcal{M}_{g}\right) .
$$

For $g<24$, the $\mathbb{Q}$-linear span of the Faber-Zagier relations is the kernel of

$$
\mathbb{Q}\left[\kappa_{1}, \kappa_{2}, \kappa_{3}, \ldots\right] \stackrel{q}{\longrightarrow} R^{*}\left(\mathcal{M}_{g}\right) \longrightarrow 0
$$

and the cycle class map $R^{*}\left(\mathcal{M}_{g}\right) \stackrel{c}{\longrightarrow} R H^{*}\left(\mathcal{M}_{g}\right)$ of question Q1 is an isomorphism.

However, the Faber-Zagier relations in genus 24 do not yield a Gorenstein ring with socle in dimension 22 ! There are too few relations in codimension 12 ,

$$
\operatorname{dim}_{\mathbb{Q}} R_{\mathrm{FZ}}^{12}\left(\mathcal{M}_{24}\right)=\operatorname{dim}_{\mathbb{Q}} R_{\mathrm{FZ}}^{10}\left(\mathcal{M}_{24}\right)+1 .
$$

\footnotetext{
${ }^{6}$ All the calculations discussed in Section 2.3 concerning the Faber-Zagier relations are by computer and were undertaken by C. Faber in the period 1991-2011 with continually improving methods. The discovery of the failure of $R_{\mathrm{FZ}}^{*}\left(\mathcal{M}_{24}\right)$ to be Gorenstein came in 2009 during a visit to Lisbon.
} 
Calculations show the Gorenstein property continues to fail (to an increasingly greater extent) as $g$ increases above 24 .

Q2. Do the Faber-Zagier relations span the ideal of relations among the $\kappa$ classes in $R^{*}\left(\mathcal{M}_{g}\right)$ for all $g$ ?

While question Q2 is completely open, a negative answer would be surprising since many different mathematical approaches have failed to find relations outside of the Faber-Zagier span [18, 40, 70, 72, 82, 87. Moreover, the Gorenstein property for the algebra of tautological classes has been proven to fail for the moduli space $\mathcal{M}_{2,8}^{\text {ct }}$ of curves of compact type and the moduli space $\overline{\mathcal{M}}_{2,20}$ of stable curves in [74, 78]. So there appears to be no compelling reason to believe the Gorenstein property holds for $\mathcal{M}_{24}$.

2.4. Hypergeometric series. The main actors in the Faber-Zagier relations are the series

$$
\begin{aligned}
& \mathrm{A}(t)=\sum_{i=0}^{\infty} \frac{(6 i) !}{(3 i) !(2 i) !} t^{i}, \\
& \mathrm{~B}(t)=\sum_{i=0}^{\infty} \frac{(6 i) !}{(3 i) !(2 i) !} \frac{6 i+1}{6 i-1} t^{i} .
\end{aligned}
$$

The functions A and B are related by the following fundamental identity observed first by Pixton [79]:

$$
\mathrm{A}(-t) \mathrm{B}(t)+\mathrm{A}(t) \mathrm{B}(-t)=-2 .
$$

The hypergeometric differential equation satisfied by $A$, written in the variable $z=288 t$, is

$$
3 z^{2} \frac{d^{2}}{d z^{2}} \mathrm{~A}+(6 z-2) \frac{d}{d z} \mathrm{~A}+\frac{5}{12} \mathrm{~A}=0 .
$$

The function $B$ is determined by $A$ and the differential equation

$$
3 z^{2} \frac{d \mathrm{~A}}{d z}+\left(\frac{z}{2}-1\right) \mathrm{A}=\mathrm{B}
$$

In the proofs of the Faber-Zagier relations, geometric sources for the series $\mathrm{A}$ and $\mathrm{B}$ were found. The two approaches [40, 71] in $R^{*}\left(\mathcal{M}_{g}\right)$ both find the series in the Frobenius geometry associated to $\mathbb{P}^{1}$. In the proof of [72] in $R H^{*}\left(\mathcal{M}_{g}\right)$, the functions $\mathrm{A}$ and $\mathrm{B}$ appear in the $R$-matrix of the Frobenius manifold associated to 3 -spin curves. 
2.5. Descendents. The series A appears in the asymptotic expansion of the Airy function related to the Witten-Kontsevich theory of descendent integration over the moduli space of stable curves.

Let $\overline{\mathcal{M}}_{g, n}$ be the moduli space of stable genus $g$ curves with $n$ marked points. The cotangent line at the $i^{\text {th }}$ marking defines a line bundle

$$
\mathbb{L}_{i} \rightarrow \overline{\mathcal{M}}_{g, n}
$$

with first Chern class

$$
\psi_{i}=c_{1}\left(\mathbb{L}_{i}\right) \in A^{1}\left(\overline{\mathcal{M}}_{g, n}\right) .
$$

The descendent integrals are defined by

$$
\left\langle\tau_{k_{1}} \tau_{k_{2}} \ldots \tau_{k_{n}}\right\rangle_{g}=\int_{\overline{\mathcal{M}}_{g, n}} \psi_{1}^{k_{1}} \psi_{2}^{k_{2}} \ldots \psi_{n}^{k_{n}} .
$$

The bracket (4) vanishes unless the dimension constraint

$$
3 g-3+n=\sum_{i=1}^{n} k_{i}
$$

is satisfied. The associated generating series in the variables $\left\{t_{i}\right\}_{i \geq 0}$ is

$$
\mathcal{F}\left(t_{0}, t_{1}, \ldots\right)=\sum_{\substack{g \geq 0, n \geq 1 \\ 2 g-2+n>0}} \frac{1}{n !} \sum_{k_{1}, \ldots, k_{n} \geq 0}\left\langle\tau_{k_{1}} \tau_{k_{2}} \ldots \tau_{k_{n}}\right\rangle_{g} t_{k_{1}} t_{k_{2}} \ldots t_{k_{n}} .
$$

Witten [88] conjectured

$$
u=\frac{\partial^{2} \mathcal{F}}{\partial t_{0}^{2}}
$$

is a solution of the KdV hierarchy. The first equations of the hierarchy are

$$
\begin{aligned}
& u_{t_{1}}=u u_{x}+\frac{1}{12} u_{x x x}, \\
& u_{t_{2}}=\frac{1}{2} u^{2} u_{x}+\frac{1}{12}\left(2 u_{x} u_{x x}+u u_{x x x}\right)+\frac{1}{240} u_{x x x x x},
\end{aligned}
$$

where we have identified $x$ here with $t_{0}$. Together with the (elementary) string equation,

$$
\frac{\partial \mathcal{F}}{\partial t_{0}}=\sum_{i=0}^{\infty} t_{i+1} \frac{\partial \mathcal{F}}{\partial t_{i}}+\frac{t_{0}^{2}}{2}
$$

Witten's conjecture uniquely determines the series $\mathcal{F}$. The conjecture was proven by Kontsevich [50]. See [46, 63, 66] for other proofs. 
A basic relationship between the descendent integrals and the A series is derived via Kontsevich's matrix integral in [8, 50]:

$$
\left.\exp (\mathcal{F})\right|_{t_{i}=-(2 i-1) ! ! \lambda^{-2 i-1}}=\mathrm{A}\left(-\frac{\lambda^{-3}}{288}\right) .
$$

For example, the terms of $\exp (\mathcal{F})$ which contribute to the $\lambda^{-3}$ coefficient are:

$$
\begin{aligned}
\left\langle\tau_{0}^{3}\right\rangle_{0} \frac{\left(-\lambda^{-1}\right)^{3}}{3 !}+\left\langle\tau_{1}\right\rangle_{1}\left(-\lambda^{-3}\right) & =\left(\frac{1}{6}+\frac{1}{24}\right)\left(-\lambda^{-3}\right) \\
& =\frac{1440}{24}\left(-\frac{\lambda^{-3}}{288}\right) \\
& =\frac{6 !}{3 ! 2 !}\left(-\frac{\lambda^{-3}}{288}\right) .
\end{aligned}
$$

The result is the $\lambda^{-3}$ term of $\mathrm{A}\left(-\frac{\lambda^{-3}}{288}\right)$.

Q3. Is the descendent evaluation (5) related to the occurrence of the $\mathrm{A}$ series in the relations among tautological classes?

Question Q3 was first raised in [8] where the many appearances of the $A$ and $B$ series related to the moduli spaces of curves are surveyed.

2.6. Markings. The algebra of tautological classes may also be studied on the moduli spaces

$$
\mathcal{M}_{g, n}, \quad \mathcal{M}_{g, n}^{\mathrm{rt}}, \quad \mathcal{C}_{g}^{n}
$$

of pointed curves lying over $\mathcal{M}_{g}$. Here, $\mathcal{M}_{g, n}$ is the moduli space of nonsingular curves with distinct markings, $\mathcal{M}_{g, n}^{\mathrm{rt}}$ is the moduli of curves with rational tails, and $\mathcal{C}_{g}^{n}$ is the $n^{\text {th }}$ fiber product of the universal curve

$$
\pi: \mathcal{C}_{g} \rightarrow \mathcal{M}_{g}
$$

In addition to the $\kappa$ classes, there are cotangent line classes $\psi_{i}$ at the markings and diagonal classes. The full algebra of tautological classes will be considered in Section 4.8 .

\section{3. $\kappa$ CLASSES ON $\mathcal{M}_{g, n}^{\text {ct }}$}

3.1. Compact type. A connected nodal curve $C$ is of compact type if the dual graph of $C$ is a tree (or, equivalently, if the Jacobian of $C$ 
is compact). The moduli space $\mathcal{M}_{g, n}^{\text {ct }}$ of curves of compact type lies in between the moduli spaces of nonsingular and stable curves

$$
\mathcal{M}_{g, n} \subset \mathcal{M}_{g, n}^{\mathrm{ct}} \subset \overline{\mathcal{M}}_{g, n} .
$$

The algebra of $\kappa$ classes on $\mathcal{M}_{g, n}^{\text {ct }}$ is remarkably well-behaved. The structure is simpler and better understood than for $\mathcal{M}_{g}$. Of course, the full algebra of tautological classes on $\mathcal{M}_{g, n}^{\text {ct }}$ contains more than the $\kappa$ classes (and will be discussed in Section 4.8).

3.2. $\kappa$ classes. The definition of the $\kappa$ classes on $\mathcal{M}_{g}$ is easily extended to the moduli of stable curves. Let

$$
\pi: \overline{\mathcal{M}}_{g, n+1} \rightarrow \overline{\mathcal{M}}_{g, n}
$$

be the universal curve viewed as the $(n+1)$-pointed space, and let

$$
\psi_{n+1}=c_{1}\left(\mathbb{L}_{n+1}\right) \in A^{1}\left(\overline{\mathcal{M}}_{g, n+1}\right)
$$

be the Chern class of the cotangent line at the last marking. The $\kappa$ classes are

$$
\kappa_{r}=\pi_{*}\left(\psi_{n+1}^{r+1}\right) \in A^{r}\left(\overline{\mathcal{M}}_{g, n}\right), \quad i \geq 0 .
$$

The simplest is $\kappa_{0}$ which equals $2 g-2+n$ times the unit in $A^{0}\left(\overline{\mathcal{M}}_{g, n}\right)$.

The restriction of $\kappa_{r} \in A^{r}\left(\overline{\mathcal{M}}_{g}\right)$ to $\mathcal{M}_{g}$ via the inclusion

$$
\mathcal{M}_{g} \subset \overline{\mathcal{M}}_{g}
$$

agrees with the definition of $\kappa_{r} \in A^{r}\left(\mathcal{M}_{g}\right)$ of Section 1.1. The $\kappa$ classes on $\mathcal{M}_{g, n}^{\text {ct }}$ are defined via restriction from $\overline{\mathcal{M}}_{g, n}$.

Define the $\kappa$ ring for curves of compact type,

$$
\kappa^{*}\left(\mathcal{M}_{g, n}^{\mathrm{ct}}\right) \subset A^{*}\left(\mathcal{M}_{g, n}^{\mathrm{ct}}\right),
$$

to be the $\mathbb{Q}$-subalgebra generated by the $\kappa$ classes. Of course, the $\kappa$ rings are graded by degree.

3.3. Basic results. The $\operatorname{ring} \kappa^{*}\left(\mathcal{M}_{g, n}^{\mathrm{ct}}\right)$ is generated over $\mathbb{Q}$ by the classes

$$
\kappa_{1}, \kappa_{2}, \ldots, \kappa_{g-1+\left\lfloor\frac{n}{2}\right\rfloor} \in \kappa^{*}\left(\mathcal{M}_{g, n}^{\mathrm{ct}}\right) .
$$

If $n>0$, there are no relations of degree less than or equal to $g-1+\left\lfloor\frac{n}{2}\right\rfloor$. In $\kappa^{*}\left(\mathcal{M}_{g}^{\text {ct }}\right)$, there are no relations of degree less than $g-1$ (whether degree $g-1$ relations can occur is not known). The proofs of the above generation and freeness results can be found in [69]. 
The nonvanishing and vanishing results for $\kappa^{*}\left(\mathcal{M}_{g, n}^{\mathrm{ct}}\right)$,

$$
\kappa^{2 g-3+n}\left(\mathcal{M}_{g, n}^{\mathrm{ct}}\right) \cong \mathbb{Q}, \quad \kappa^{>2 g-3+n}\left(\mathcal{M}_{g, n}^{\mathrm{ct}}\right)=0,
$$

parallel to (2) for the $\kappa$ classes on $\mathcal{M}_{g}$, were proven in [21, 34]. The proportionalities in $\kappa^{2 g-3+n}\left(\mathcal{M}_{g, n}^{\text {ct }}\right)$ of the monomials in the $\kappa$ classes are determined by Hodge integral evaluations [19, 20, 31].

3.4. Universality. A new and surprising feature about the $\kappa$ rings in the compact type case is the following universality result proven in [69]. There appears to be no parallel for $\mathcal{M}_{g}$.

Theorem 2 (P. 2009). Let $g>0$ and $n>0$, then the assignment $\kappa_{i} \mapsto \kappa_{i}$ extends to a ring isomorphism

$$
\iota: \kappa^{*}\left(\mathcal{M}_{g-1, n+2}^{\mathrm{ct}}\right) \cong \kappa^{*}\left(\mathcal{M}_{g, n}^{\mathrm{ct}}\right) .
$$

In other words, the relations among the $\kappa$ classes in the above cases are genus independent! By composing the isomorphisms $\iota$ of Theorem 2 , we obtain isomorphisms

$$
\iota: \kappa^{*}\left(\mathcal{M}_{0,2 g+n}^{\mathrm{ct}}\right) \cong \kappa^{*}\left(\mathcal{M}_{g, n}^{\mathrm{ct}}\right)
$$

so long as $n>0$. Hence, universality reduces all questions about the $\kappa$ rings to genus 0 . Calculations of the relations, bases, and Betti numbers of the ring $\kappa^{*}\left(\mathcal{M}_{g, n>0}^{\mathrm{ct}}\right)$ are obtained in [69] using the genus 0 reduction.

Let $P(d)$ be the set of partitions of $d$, and let

$$
P(d, k) \subset P(d)
$$

be the set of partitions of $d$ into at most $k$ parts. Let $|P(d, k)|$ be the cardinality. To a partition with positive parts $\mathbf{p}=\left(p_{1}, \ldots, p_{\ell}\right)$ in $P(d, k)$, we associate a $\kappa$ monomial by

$$
\kappa_{\mathbf{p}}=\kappa_{p_{1}} \cdots \kappa_{p_{\ell}} \in \kappa^{d}\left(\mathcal{M}_{g, n}^{\mathrm{ct}}\right) .
$$

Theorem 3 (P. 2009). For $n>0$, a $\mathbb{Q}$-basis of $\kappa^{d}\left(\mathcal{M}_{g, n}^{\text {ct }}\right)$ is given by

$$
\left\{\kappa_{\mathbf{p}} \mid \mathbf{p} \in P(d, 2 g-2+n-d)\right\} \text {. }
$$

The Betti number calculation,

$$
\operatorname{dim}_{\mathbb{Q}} \kappa^{d}\left(\mathcal{M}_{g, n}^{\mathrm{ct}}\right)=|P(d, 2 g-2+n-d)|,
$$

is implied by Theorem 3 . 
Q4. Is there a representation theoretic formula for the multiplication of $\kappa^{*}\left(\mathcal{M}_{g, n}^{\mathrm{ct}}\right)$ in the basis of Theorem 3 ?

Question Q4 appears directly parallel to questions in the Schubert calculus. Recent progress on Q4 has been made by Setayesh [83] who has found a formula involving the combinatorics of partitions.

Theorems 2 and 3 for the $\kappa$ rings in the compact type case require at least 1 marked point. In the unmarked case, half of the universality still holds. The assignment $\kappa_{i} \mapsto \kappa_{i}$ extends to a surjection

$$
\iota_{g}: \kappa^{*}\left(\mathcal{M}_{0,2 g}^{\mathrm{ct}}\right) \rightarrow \kappa^{*}\left(\mathcal{M}_{g}^{\mathrm{ct}}\right) .
$$

However, a nontrivial kernel is possible. The first kernel occurs in genus $g=5$.

Q5. What is the kernel of $\kappa^{*}\left(\mathcal{M}_{0,2 g}^{\mathrm{ct}}\right) \rightarrow \kappa^{*}\left(\mathcal{M}_{g}^{\mathrm{ct}}\right)$ ?

In genus 5 , the kernel of $\iota_{5}$ is related to Getzler's relation in $\overline{\mathcal{M}}_{1,4}$, see [69] for a discussion. A complete answer to Q5 will likely involve sequences of special relations in the tautological ring.7

The proofs of Theorems 2 and 3 were obtained in [69] by studying the virtual class of the moduli space of stable quotients [60]. The results were precursors to the proof [70, 171] of the Faber-Zagier relations via the moduli of stable quotients.

3.5. Relations. A natural question to ask is whether $\kappa$ relations in the compact type case can be put in a form parallel to Theorem 1 . An answer has been found by Pixton [80, Section 3.4].

We define a set of relations as follows. Let

$$
\mathbf{p}=\left\{p_{1}, p_{2}, p_{3}, \ldots\right\}
$$

be a variable set indexed by all positive integers. Let

$$
\begin{aligned}
\Psi(t, \mathbf{p})=\left(1+t p_{2}+t^{2} p_{4}\right. & \left.+t^{3} p_{6}+\ldots\right) \sum_{i=0}^{\infty}(2 i-1) ! ! t^{i} \\
& +\left(p_{1}+t p_{3}+t^{2} p_{5}+\ldots\right),
\end{aligned}
$$

\footnotetext{
7 Q5 is perhaps the narrowest question discussed in the paper, but I am very curious to know the answer.
} 
where $(2 i-1) ! !=\frac{(2 i) !}{2^{i} i !}$ as usual. Define the constants $C_{r}^{\mathrm{P}}(\sigma)$ by the formula

$$
\log (\Psi)=\sum_{\sigma} \sum_{r=0}^{\infty} C_{r}^{\mathrm{P}}(\sigma) t^{r} \mathbf{p}^{\sigma} .
$$

Here, $\sigma$ denotes any partition with positive parts. Let

$$
\gamma^{\mathrm{P}}=\sum_{\sigma} \sum_{r=0}^{\infty} C_{r}^{\mathrm{P}}(\sigma) \kappa_{r} t^{r} \mathbf{p}^{\sigma} .
$$

Theorem 4 (Pixton 2013). In $\kappa^{d}\left(\mathcal{M}_{g, n}^{\text {ct }}\right)$, the relation

$$
\left[\exp \left(-\gamma^{\mathrm{P}}\right)\right]_{t^{d} \mathbf{p}^{\sigma}}=0
$$

holds when $d>\frac{2 g-2+n+|\sigma|}{2}$.

Theorems 2 and 3 determine the complete set of relations among the $\kappa$ classes in the $n>0$ case. Using Theorems 2 and 3 , Pixton [80] has proven that the $\mathbb{Q}$-linear span of the relations of Theorem 4 is the complete set in the $n>0$ case.

\section{Pixton's Relations on $\overline{\mathcal{M}}_{g, n}$}

4.1. Overview. Tautological classes on the moduli space $\overline{\mathcal{M}}_{g, n}$ of stable curves are obtained from $\kappa$ classes, $\psi$ classes, and the classes of boundary strata (indexed by stable graphs) 8 Decorated stable graphs provide a language for describing all tautological classes. A parallel role is played by the language of partitions in the Schubert calculus of the Grassmannian. After a brief discussion of stable graphs in Section 4.2 , the algebra of tautological classes

$$
R^{*}\left(\overline{\mathcal{M}}_{g, n}\right) \subset A^{*}\left(\overline{\mathcal{M}}_{g, n}\right)
$$

is defined in Section 4.3 .

The main recent advance is the set of relations in $R^{*}\left(\overline{\mathcal{M}}_{g, n}\right)$ found by Pixton. Pixton's relations [79] were conjectured in 2012 and first proven 72. to hold in $R H^{*}\left(\overline{\mathcal{M}}_{g, n}\right)$ in 2013 using Witten's 3-spin class ${ }^{9}$ and the Givental-Teleman classification of Cohomological Field Theories [86]. Shortly afterwards (also in 2013), Janda [39] found a proof in $R^{*}\left(\overline{\mathcal{M}}_{g, n}\right)$

\footnotetext{
${ }^{8} \mathrm{~A}$ study of the algebra of $\kappa$ classes can also be pursued on $\overline{\mathcal{M}}_{g, n}$, see [15, 16.

${ }^{9}$ Tautological relations obtained from Witten's $r$-spin class for higher $r$ are studied in [73. By Janda's result [41, the relations of [73] are contained in Pixton's set.
} 
using a mix of virtual localization 32 and $R$-matrix techniques for the equivariant stable quotients theory of $\mathbb{P}^{1}$. His argument combined elements of the Chow results of [70, 71] and the CohFT methods of [72]. A second proof in Chow via the equivariant Gromov-Witten theory of $\mathbb{P}^{1}$ was found by Janda [42] in 2015.

Pixton has further conjectured that all relations among tautological classes are obtained from his set. The claim is open and is perhaps the most important question in the subject. Pixton's relations for $R^{*}\left(\overline{\mathcal{M}}_{g, n}\right)$ specialize to the Faber-Zagier relations when restricted to the moduli space $\mathcal{M}_{g}$ of nonsingular curves.

4.2. Boundary strata. The boundary strata of the moduli space $\overline{\mathcal{M}}_{g, n}$ parameterizing complex structures on curves of fixed topological type correspond to stable graphs. The idea here is simple, but the notation requires some care.

A stable graph $\Gamma$ consists of the data

$$
\Gamma=\left(\mathrm{V}, \mathrm{H}, \mathrm{L}, \mathrm{g}: \mathrm{V} \rightarrow \mathbb{Z}_{\geq 0}, v: \mathrm{H} \rightarrow \mathrm{V}, \iota: \mathrm{H} \rightarrow \mathrm{H}\right)
$$

which satisfies the following properties:

(i) $\mathrm{V}$ is a vertex set with a genus function $\mathrm{g}: \mathrm{V} \rightarrow \mathbb{Z}_{\geq 0}$,

(ii) $\mathrm{H}$ is a half-edge set equipped with a vertex assignment

$$
v: \mathrm{H} \rightarrow \mathrm{V}
$$

and an involution $\iota: \mathrm{H} \rightarrow \mathrm{H}$,

(iii) E, the edge set, is defined by the 2-cycles of $\iota$ in $\mathrm{H}$ (self-edges at vertices are permitted),

(iv) L, the set of legs, is defined by the fixed points of $\iota$ and is endowed with a bijective correspondence with the set of markings

$$
\mathrm{L} \leftrightarrow\{1, \ldots, n\}
$$

(v) the pair $(\mathrm{V}, \mathrm{E})$ defines a connected graph,

(vi) for each vertex $v$, the stability condition holds:

$$
2 \mathrm{~g}(v)-2+\mathrm{n}(v)>0
$$

where $\mathrm{n}(v)$ is the valence of $\Gamma$ at $v$ including both edges and legs. 
An automorphism of $\Gamma$ consists of automorphisms of the sets $\mathrm{V}$ and $\mathrm{H}$ which leave invariant the structures g, $\iota$, and $v$ (and hence respect $\mathrm{E}$ and $\mathrm{L})$. Let $\operatorname{Aut}(\Gamma)$ denote the automorphism group of $\Gamma$.

The genus of a stable graph $\Gamma$ is defined by

$$
\mathrm{g}(\Gamma)=\sum_{v \in V} \mathrm{~g}(v)+h^{1}(\Gamma)
$$

A boundary stratum of the moduli space $\overline{\mathcal{M}}_{g, n}$ naturally determines a stable graph of genus $g$ with $n$ legs by considering the dual graph of a generic pointed curve parameterized by the stratum.

To each stable graph $\Gamma$, we associate the moduli space

$$
\overline{\mathcal{M}}_{\Gamma}=\prod_{v \in \mathrm{V}} \overline{\mathcal{M}}_{\mathrm{g}(v), \mathrm{n}(v)}
$$

Let $\pi_{v}$ denote the projection from $\overline{\mathcal{M}}_{\Gamma}$ to $\overline{\mathcal{M}}_{\mathrm{g}(v), \mathrm{n}(v)}$ associated to the vertex $v$. There is a canonical morphism

$$
\xi_{\Gamma}: \overline{\mathcal{M}}_{\Gamma} \rightarrow \overline{\mathcal{M}}_{g, n}
$$

with image $\AA^{10}$ equal to the closure of the boundary stratum associated to the graph $\Gamma$. To construct $\xi_{\Gamma}$, a family of stable pointed curves over $\overline{\mathcal{M}}_{\Gamma}$ is required. Such a family is easily defined by attaching the pull-backs of the universal families over each of the $\overline{\mathcal{M}}_{\mathrm{g}(v), \mathrm{n}(v)}$ along the sections corresponding to half-edges. Let

$$
[\Gamma] \in A^{*}\left(\overline{\mathcal{M}}_{g, n}\right)
$$

denote the push-forward under $\xi_{\Gamma}$ of the fundamental class of $\overline{\mathcal{M}}_{\Gamma}$.

Two examples of boundary strata in $\overline{\mathcal{M}}_{3,3}$ and their associated stable graphs are given in the following diagram.

\footnotetext{
10 The degree of $\xi_{\Gamma}$ is $|\operatorname{Aut}(\Gamma)|$.
} 

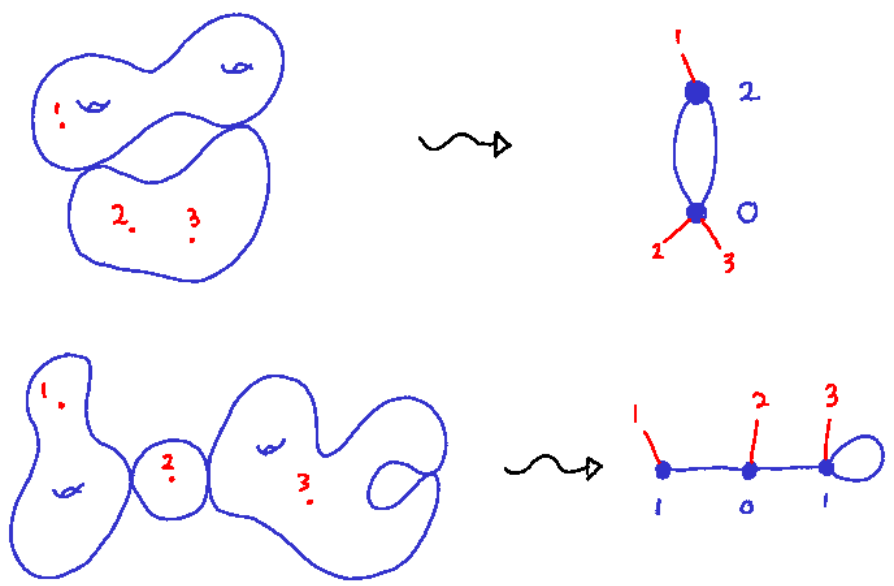

Let $\Phi$ and $\widehat{\Phi}$ be the stable graphs in the first and second cases in the diagram. The moduli space $\overline{\mathcal{M}}_{\Phi}$ in the first case is

$$
\overline{\mathcal{M}}_{0,4} \times \overline{\mathcal{M}}_{2,3}
$$

with $|\operatorname{Aut}(\Phi)|=2$. The nontrivial automorphism arises from switching the edges. The moduli space $\overline{\mathcal{M}}_{\widehat{\Phi}}$ in the second case is

$$
\overline{\mathcal{M}}_{1,2} \times \overline{\mathcal{M}}_{0,3} \times \overline{\mathcal{M}}_{1,4}
$$

with $|\operatorname{Aut}(\widehat{\Phi})|=2$. The nontrivial automorphism arises from switching the half-edges on the self-edge.

4.3. Strata algebra. Let $\mathcal{S}_{g, n}^{*}$ be the $\mathbb{Q}$-algebra of $\kappa$ and $\psi$ classes supported on the strata of $\overline{\mathcal{M}}_{g, n}$. A $\mathbb{Q}$-basis of $\mathcal{S}_{g, n}^{*}$ is given by isomorphism classes of pairs $[\Gamma, \gamma]$ where $\Gamma$ is a stable graph corresponding to a stratum of the moduli space,

$$
\overline{\mathcal{M}}_{\Gamma} \rightarrow \overline{\mathcal{M}}_{g, n}
$$

and $\gamma$ is a product of $\kappa$ and $\psi$ classes on $\overline{\mathcal{M}}_{\Gamma}$. The $\kappa$ classes are associated to the vertices, and the $\psi$ classes are associated to the halfedges. The only condition imposed is that the degrees of the $\kappa$ and $\psi$ classes associated to a vertex $v \in \mathrm{V}(\Gamma)$ together do not exceed the dimension $3 \mathrm{~g}(v)-3+\mathrm{n}(v)$ of the moduli space at $v$.

For the graph $\Phi$ associated to a stratum of $\overline{\mathcal{M}}_{3,3}$ in the diagram, let $v_{0}$ and $v_{2}$ denote the vertices of genus 0 and 2 respectively. Let the left edge consist of the two half edges $h_{0}-h_{2}$ where $h_{0}$ is incident to $v_{0}$ 
and $h_{2}$ is incident to $v_{2}$. Then,

$$
\left[\Phi, \kappa_{1}\left[v_{0}\right] \kappa_{2}\left[v_{2}\right] \psi_{h_{2}}^{2} \psi_{1}\right]
$$

is an example of such a pair. The codimension of the pair (7) is

$$
8=2+6
$$

2 for the nodes of $\Phi$ and 6 for the $\kappa$ and $\psi$ classes.

The strata algebra $\mathcal{S}_{g, n}^{*}$ is graded by codimension

$$
\mathcal{S}_{g, n}^{*}=\bigoplus_{d=0}^{3 g-3+n} \mathcal{S}_{g, n}^{d}
$$

and carries a product for which the natural push-forward map

$$
\mathcal{S}_{g, n}^{*} \rightarrow A^{*}\left(\overline{\mathcal{M}}_{g, n}\right)
$$

is a ring homomorphism, see [72, Section 0.3] for a detailed discussion.

The image of (8) is, by definition, the tautological ring ${ }^{11}$

$$
R^{*}\left(\overline{\mathcal{M}}_{g, n}\right) \subset A^{*}\left(\overline{\mathcal{M}}_{g, n}\right) .
$$

Hence, we have a quotient

$$
\mathcal{S}_{g, n}^{*} \stackrel{q}{\longrightarrow} R^{*}\left(\overline{\mathcal{M}}_{g, n}\right) \longrightarrow 0
$$

The ideal of tautological relations is the kernel of $q$.

In the strata algebra, the basis elements $[\Gamma, \gamma]$ are treated formally. In the case $(g, n)=(0,4)$, we have

$$
\operatorname{dim}_{\mathbb{Q}} \mathcal{S}_{0,4}^{0}=1, \quad \operatorname{dim}_{\mathbb{Q}} \mathcal{S}_{0,4}^{1}=8 .
$$

Let $\Gamma$. be the unique graph of genus 0 with 4 markings and a single vertex $v$. Then

$$
[\Gamma \bullet, 1] \in \mathcal{S}_{0,4}^{0}
$$

is a basis. In codimension 1 , the 5 possibilities for $\gamma$ on $\Gamma$ • yield the pairs

$$
\left[\Gamma_{\bullet}, \kappa_{1}[v]\right],\left[\Gamma_{\bullet}, \psi_{1}\right],\left[\Gamma_{\bullet}, \psi_{2}\right],\left[\Gamma_{\bullet}, \psi_{3}\right],\left[\Gamma_{\bullet}, \psi_{4}\right] \in \mathcal{S}_{0,4}^{1}
$$

In addition, there are 3 pairs

$$
\left[\Gamma_{1,2 \mid 3,4}, 1\right],\left[\Gamma_{1,3 \mid 2,4}, 1\right],\left[\Gamma_{1,4 \mid 2,3}, 1\right] \in \mathcal{S}_{0,4}^{1}
$$

\footnotetext{
${ }^{11}$ Our definition here follows the Appendix of [33]. See [22, Section 1] for a more intrinsic approach.
} 
where the underlying graphs have 2 vertices (and correspond to the usual boundary strata). The kernel

$$
\mathcal{S}_{0,4}^{1} \stackrel{q}{\longrightarrow} R^{1}\left(\overline{\mathcal{M}}_{0,4}\right)
$$

is 7 dimensional and contains the basic linear equivalence of the three boundary divisors of

$$
\overline{\mathcal{M}}_{0,4} \cong \mathbb{P}^{1}
$$

see [47, 52] for a study of $R^{*}\left(\overline{\mathcal{M}}_{0, n}\right)$.

The first geometrically interesting relation ${ }^{12}$ was found in genus 1 by Getzler [28] in 1996. Soon after, several low genus relations were determined. Below is a tautological relation ${ }^{13}$ in codimension 2 on $\overline{\mathcal{M}}_{2,3}$ found in [6] in 1998.

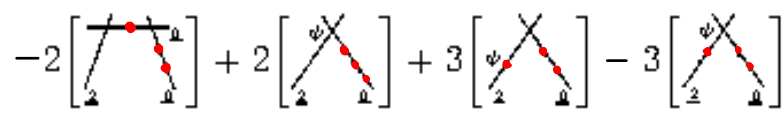

$+\frac{2}{5}\left[\begin{array}{ll}1 & 1\end{array}\right]-\frac{6}{5}\left[\begin{array}{ll}1 & 0 \\ 1 & 1\end{array}\right]+\frac{12}{5}\left[\begin{array}{ll}1 & 1 \\ 1 & 1\end{array}\right]-\frac{18}{5}\left[\begin{array}{ll}1 & 1 \\ 1 & 1\end{array}\right]-\frac{6}{5}\left[\begin{array}{ll}1 & 1 \\ 1 & 1\end{array}\right]$

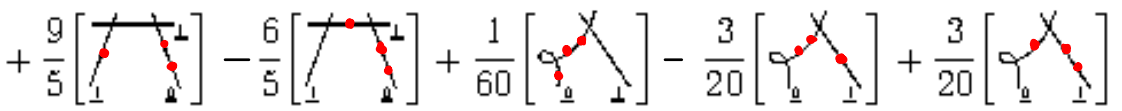

$-\frac{1}{60}\left[\begin{array}{ll}X_{2} & X_{1}\end{array}\right]+\frac{1}{5}\left[\begin{array}{l}X_{1} \\ X_{2}\end{array}\right]-\frac{3}{5}\left[\begin{array}{l}X_{1} \\ X_{2}\end{array}\right]+\frac{1}{5}\left[\begin{array}{l}X_{1} \\ X_{2}\end{array}\right]-\frac{1}{10}\left[Y_{1} X_{2}\right]-\frac{1}{10}\left[X_{1} X_{1}\right]=0$

Pixton's set puts order to the chaos of strata classes and coefficients which appear in the above relation (and in all the other relations ${ }^{14}$ found in the period after Getzler's discovery).

4.4. Vertex, leg, and edge factors. Pixton's relations are determined by a set

$$
\mathcal{P}=\left\{\mathcal{R}_{g, A}^{d}\right\}
$$

of elements $\mathcal{R}_{g, A}^{d} \in \mathcal{S}_{g, n}^{d}$ associated to the data

\footnotetext{
${ }^{12} \mathrm{~A}$ proof of Getzler's relation in Chow was given later in 67.

${ }^{13}$ The strata classes in the genus 2 relation of the diagram have been represented by their topological type instead of their associated dual graph. The genera of the components are underlined. The red marked points are unlabeled. Each picture represents the sum of the 6 possible labelings of the markings. The diagram (taken from [6]) was typeset by P. Belorousski.

${ }^{14}$ For example, see [30, 48, 49] for further relations in genus 2 and 3.
} 
- $g, n \in \mathbb{Z}_{\geq 0}$ in the stable range $2 g-2+n>0$,

- $A=\left(a_{1}, \ldots, a_{n}\right), \quad a_{i} \in\{0,1\}$

- $d \in \mathbb{Z}_{\geq 0}$ satisfying $d>\frac{g-1+\sum_{i=1}^{n} a_{i}}{3}$.

The elements $\mathcal{R}_{g, A}^{d}$ are expressed as sums over stable graphs of genus $g$ with $n$ legs. Before writing the formula for $\mathcal{R}_{g, A}^{d}$, a few definitions are required.

Definitions of $H_{0}$ and $H_{1}$. The hypergeometric series $A$ and $B$ of Section 2.4 enter Pixton's relations in following form:

$$
\begin{aligned}
& \mathrm{H}_{0}(T)=\mathrm{A}(-T)=\sum_{i=0}^{\infty} \frac{(6 i) !}{(2 i) !(3 i) !}(-T)^{i}=1-60 T+27720 T^{2}-\cdots \\
& \mathrm{H}_{1}(T)=-\mathrm{B}(-T)=-\sum_{i=0}^{\infty} \frac{(6 i) !}{(2 i) !(3 i) !} \frac{6 i+1}{6 i-1}(-T)^{i}=1+84 T-32760 T^{2}+\cdots
\end{aligned}
$$

Definition of $\kappa(f)$. Let $f(T)$ be a power series with vanishing constant and linear terms,

$$
f(T) \in T^{2} \mathbb{Q}[[T]] .
$$

For each $\overline{\mathcal{M}}_{g, n}$, we define

$$
\kappa(f)=\sum_{m \geq 0} \frac{1}{m !} p_{m *}\left(f\left(\psi_{n+1}\right) \cdots f\left(\psi_{n+m}\right)\right) \in A^{*}\left(\overline{\mathcal{M}}_{g, n}\right),
$$

where $p_{m}$ is the forgetful map

$$
p_{m}: \overline{\mathcal{M}}_{g, n+m} \rightarrow \overline{\mathcal{M}}_{g, n}
$$

By the vanishing in degrees 0 and 1 of $f$, the sum $(9)$ is finite.

Definitions of $\mathrm{G}_{g, n}$ and $\zeta_{v}$. Let $\mathrm{G}_{g, n}$ be the (finite) set of stable graphs of genus $g$ with $n$ legs (up to isomorphism). Let $\Gamma \in \mathrm{G}_{g, n}$. For each vertex $v \in \mathrm{V}$, we introduce an auxiliary variable $\zeta_{v}$ and impose the conditions

$$
\zeta_{v} \zeta_{v^{\prime}}=\zeta_{v^{\prime}} \zeta_{v}, \quad \zeta_{v}^{2}=1
$$

The variables $\zeta_{v}$ will be responsible for keeping track of a local parity condition at each vertex. 
The formula for $\mathcal{R}_{g, A}^{d}$ is a sum over $\mathrm{G}_{g, n}$. The summand corresponding to $\Gamma \in \mathrm{G}_{g, n}$ is a product of vertex, leg, and edge factors:

- For $v \in \mathrm{V}$, let $\kappa_{v}=\kappa\left(T-T \mathrm{H}_{0}\left(\zeta_{v} T\right)\right)$.

- For $l \in \mathrm{L}$, let $\mathrm{H}_{l}=\zeta_{v(l)}^{a_{l}} \mathrm{H}_{a_{l}}\left(\zeta_{v(l)} \psi_{l}\right)$, where $v(l) \in V$ is the vertex to which the leg is assigned.

- For $e \in \mathrm{E}$, let

$$
\begin{aligned}
\Delta_{e} & =\frac{\zeta^{\prime}+\zeta^{\prime \prime}-\mathrm{H}_{0}\left(\zeta^{\prime} \psi^{\prime}\right) \zeta^{\prime \prime} \mathrm{H}_{1}\left(\zeta^{\prime \prime} \psi^{\prime \prime}\right)-\zeta^{\prime} \mathrm{H}_{1}\left(\zeta^{\prime} \psi^{\prime}\right) \mathrm{H}_{0}\left(\zeta^{\prime \prime} \psi^{\prime \prime}\right)}{\psi^{\prime}+\psi^{\prime \prime}} \\
& =\left(60 \zeta^{\prime} \zeta^{\prime \prime}-84\right)+\left[32760\left(\zeta^{\prime} \psi^{\prime}+\zeta^{\prime \prime} \psi^{\prime \prime}\right)-27720\left(\zeta^{\prime} \psi^{\prime \prime}+\zeta^{\prime \prime} \psi^{\prime}\right)\right]+\cdots,
\end{aligned}
$$

where $\zeta^{\prime}, \zeta^{\prime \prime}$ are the $\zeta$-variables assigned to the vertices adjacent to the edge $e$ and $\psi^{\prime}, \psi^{\prime \prime}$ are the $\psi$-classes corresponding to the half-edges.

The numerator of $\Delta_{e}$ is divisible by the denominator due to the identity 15

$$
\mathrm{H}_{0}(T) \mathrm{H}_{1}(-T)+\mathrm{H}_{0}(-T) \mathrm{H}_{1}(T)=2 .
$$

Certainly, $\Delta_{e}$ is symmetric in the half-edges.

4.5. Pixton's relations $\mathcal{P}$. Let $A=\left(a_{1}, \ldots, a_{n}\right) \in\{0,1\}^{n}$. Let

$$
\mathcal{R}_{g, A}^{d} \in \mathcal{S}_{g, n}^{d}
$$

be the degree $d$ component of the strata algebra class

$$
\sum_{\Gamma \in \mathrm{G}_{g, n}} \frac{1}{|\operatorname{Aut}(\Gamma)|} \frac{1}{2^{h^{1}(\Gamma)}}\left[\Gamma,\left[\prod \kappa_{v} \prod \mathrm{H}_{l} \prod \Delta_{e}\right]_{\prod_{v} \zeta_{v}^{\mathrm{g}(v)-1}}\right] \in \mathcal{S}_{g, n}^{*},
$$

where the products are taken over all vertices, all legs, and all edges of the graph $\Gamma$. The subscript $\prod_{v} \zeta_{v}^{\mathrm{g}(v)-1}$ indicates the coefficient of the monomial $\prod_{v} \zeta_{v}^{\mathrm{g}(v)-1}$ after the product inside the brackets is expanded. In fact,

$$
\mathcal{R}_{g, A}^{d}=0 \in \mathcal{S}_{g, n}^{d}
$$

unless the parity constraint

$$
g \equiv d+1+\sum_{i=1}^{n} a_{i} \bmod 2
$$

holds.

\footnotetext{
${ }^{15}$ The identity is equivalent to 3 .
} 
We denote by $\mathcal{P}$ the set of classes $\mathcal{R}_{g, A}^{d}$ where

$$
d>\frac{g-1+\sum_{i=1}^{n} a_{i}}{3}
$$

By the following result, Pixton's set $\mathcal{P}$ consists of tautological relations. Theorem 5 (Janda 2013). Every element $\mathcal{R}_{g, A}^{d} \in \mathcal{P}$ lies in the kernel of the homomorphism

$$
q: \mathcal{S}_{g, n}^{*} \rightarrow A^{*}\left(\overline{\mathcal{M}}_{g, n}\right)
$$

4.6. Pixton's relations $\overline{\mathcal{P}}$. The set $\mathcal{P}$ is extended to a larger set

$$
\mathcal{P} \subset \overline{\mathcal{P}}
$$

of tautological relations by the following construction.

The first step is to define a tautological relation $\mathcal{R}_{g, A, \sigma}^{d} \in \mathcal{S}_{g, n}^{d}$ associated to the data

- $g, n \in \mathbb{Z}_{\geq 0}$ in the stable range $2 g-2+n>0$,

- $A=\left(a_{1}, \ldots, a_{n}\right), a_{i} \in \mathbb{Z}_{\geq 0}, \quad a_{i} \equiv 0$ or $1 \bmod 3$,

- $\sigma$ is a partition of size $|\sigma|$ with parts $\sigma_{i} \equiv 0$ or $1 \bmod 3$,

- $d \in \mathbb{Z}_{\geq 0}$ satisfying $d>\frac{g-1+\sum_{i=1}^{n} a_{i}+|\sigma|}{3}$.

Let $B=\left(b_{1}, \ldots, b_{n}, b_{n+1}, \ldots, b_{n+\ell}\right)$ be the unique vector satisfying

$$
b_{j} \in\{0,1\} \text { and }\left\{\begin{array}{lll}
b_{j} \equiv a_{j} & \bmod 3 & 1 \leq j \leq n \\
b_{j} \equiv \sigma_{j-n} & \bmod 3 & n+1 \leq j \leq n+\ell
\end{array},\right.
$$

where $\ell$ is the length of $\sigma$. Let

$$
\widehat{d}=d-\sum_{j=1}^{n} \frac{a_{j}-b_{j}}{3}-\sum_{j=n+1}^{n+\ell} \frac{\sigma_{j-n}-b_{j}}{3}>\frac{g-1+\sum_{j=1}^{n+\ell} b_{j}}{3}
$$

Pixton's definition ${ }^{16}$ of $\mathcal{R}_{g, A, \sigma}^{d} \in \mathcal{S}_{g, n}^{d}$ is

$$
\mathcal{R}_{g, A, \sigma}=p_{\ell *}\left(\mathcal{R}_{g, B}^{\widehat{d}} \cdot \prod_{j=1}^{n} \psi_{j}^{\frac{a_{j}-b_{j}}{3}} \prod_{j=n+1}^{n+\ell} \psi_{j}^{1+\frac{\sigma_{j-n}-b_{j}}{3}}\right),
$$

\footnotetext{
${ }^{16}$ Our conventions here differ from 79 by a global sign.
} 
where $\mathcal{R}_{g, B}^{\widehat{d}} \in \mathcal{S}_{g, n+\ell}^{\widehat{d}}$ is in the set $\mathcal{P}$ and $p_{\ell *}$ is push-forward by the map forgetting the last $\ell$ markings,

$$
p_{\ell *}: \mathcal{S}_{g, n+\ell}^{*} \rightarrow \mathcal{S}_{g, n}^{*} \text {. }
$$

By Theorem 5. $\mathcal{R}_{g, B}^{\widehat{d}}$ is a tautological relation. Therefore, $\mathcal{R}_{g, A, \sigma}^{d}$ is also a tautological relation.

When $A=\emptyset$, the relations $\mathcal{R}_{g, \sigma}^{d} \in \mathcal{S}_{g, 0}^{d}$ yield, after restriction to

$$
\mathcal{M}_{g} \subset \overline{\mathcal{M}}_{g},
$$

the Faber-Zagier relations of Theorem $1{ }^{17}$

Pixton's set $\overline{\mathcal{P}}$ is obtained by taking the closure of the extended set of classes

$$
\left\{\mathcal{R}_{g, A, \sigma}^{d} \in \mathcal{S}_{g, n}^{d} \mid d>\frac{g-1+\sum_{i=1}^{n} a_{i}+|\sigma|}{3}\right\}
$$

under push-forward by all boundary maps: add to the set (10) all classes in $\mathcal{S}_{g, n}^{*}$ which are obtained from a stable graph

$$
\Gamma \in \mathrm{G}_{g, n}
$$

with a class $\mathcal{R}_{g(v), A(v), \sigma(v)}^{d(v)}$ placed on a single vertex $v \in \mathrm{V}(\Gamma)$ and any product of tautological classes placed on the other vertices of $\Gamma$. By Theorem 5, every class in $\overline{\mathcal{P}}$ determines a tautological relation.

The subset $\overline{\mathcal{P}}$ lying in a fixed $\mathcal{S}_{g, n}^{d}$ is effectively computable. By the dimension restriction

$$
d>\frac{g-1+\sum_{i=1}^{n} a_{i}+|\sigma|}{3},
$$

only finitely many $\mathcal{R}_{g, A, \sigma}^{d}$ lie in $\overline{\mathcal{P}}$. The closure process by boundary push-forward is again finite because of the dimension restriction. Hence, the $\mathbb{Q}$-linear span of $\overline{\mathcal{P}}$ in $\mathcal{S}_{g, n}^{d}$ is generated by a finite list of explicit classes.

Why stop at $\overline{\mathcal{P}}$ ? Why not consider the closure with respect to further push-forwards and pull-backs via the standard boundary and forgetful

${ }^{17}$ The relation $\mathcal{R}_{g, \sigma, A}^{d}$ is trivial unless the parity condition

$$
g \equiv d+|\sigma|+1+\sum_{i=1}^{n} a_{i} \quad \bmod 2
$$

holds. 
maps? Pixton [79, 80] has proven the set $\overline{\mathcal{P}}$ is closed under all these further operations ${ }^{18}$

Q6. Do Pixton's relations $\overline{\mathcal{P}}$ span the ideal of relations among the tautological classes in $R^{*}\left(\overline{\mathcal{M}}_{g, n}\right)$ for all $g$ and $n$ ?

4.7. Pixton's conjecture. Pixton has conjectured an affirmative answer to question Q6. The evidence for Pixton's conjecture is (at least) the following:

(i) All the previously found relations occur in $\overline{\mathcal{P}}$. The theory in genus 0 is straightforward (and explained in [72, Section 3.6]). Modulo simpler relations, Getzler's genus 1 relation is

$$
\mathcal{R}_{1,(1,1,1,1)}^{2} \in \mathcal{S}_{1,4}^{2} .
$$

Pixton's conjecture is true ${ }^{19}$ for $g \in\{0,1\}$ and all $n$. The genus 2 relation displayed in Section 4.3 is

$$
\mathcal{R}_{2,(1,1,1)}^{2} \in \mathcal{S}_{2,3}^{2}
$$

modulo simpler relations.

(ii) Computer calculations of Pixton's relations for low $(g, n)$ often yield Gorenstein rings (forbidding further relations). However, just as in the Faber-Zagier case, Pixton's relations do not always yield Gorenstein rings.

(iii) Janda [41] has proven that a wide class of semisimple Cohomological Field Theories (including higher projective spaces and $r$-spin curves) will not yield relations outside of $\overline{\mathcal{P}}$.

Question Q6 has not been investigated as extensively as question Q2 for the Faber-Zagier relations since the moduli spaces of stable curves are computationally more difficult to handle. However, the failure to

\footnotetext{
${ }^{18}$ From Pixton's results, the most efficient definition of the $\mathbb{Q}$-linear span of $\overline{\mathcal{P}}$ is as the smallest set of ideals

$$
\left\{\mathcal{I}_{g, n} \subset \mathcal{S}_{g, n}^{*}\right\}
$$

which contains $\mathcal{P}$ and is closed under the natural boundary and forgetful operations.

${ }^{19}$ The proof uses the Gorenstein property of the tautological rings $R^{*}\left(\overline{\mathcal{M}}_{0, n}\right)$ and $R^{*}\left(\overline{\mathcal{M}}_{1, n}\right)$. The Gorenstein property is clear in $g=0$ since

$$
R^{*}\left(\overline{\mathcal{M}}_{0, n}\right) \cong H^{*}\left(\overline{\mathcal{M}}_{0, n}\right)
$$
}

and is proven in $g=1$ in $[75]$. 
find additional $\kappa$ relations in $R^{*}\left(\mathcal{M}_{g}\right)$ may also be viewed as supporting Q6 by the restriction property.

Pixton's proposal provides an effective calculus of tautological classes on the moduli spaces $\overline{\mathcal{M}}_{g, n}$ of stable curves. With an affirmative answer to Q6, Pixton's calculus provides a complete answer. Perhaps a reformulation of the set $\overline{\mathcal{P}}$ in a more directly algebraic setting will eventually be found. How the subject will develop depends very much on the answer to Q6.

Even a few years ago, a calculus for the moduli space of curves seemed far out of reach. Pixton's proposal has led to a striking change of outlook.

4.8. Nonsingular and compact type curves. The moduli spaces

$$
\mathcal{M}_{g, n}, \mathcal{M}_{g, n}^{\mathrm{rt}}, \mathcal{M}_{g, n}^{\mathrm{ct}} \subset \overline{\mathcal{M}}_{g, n}
$$

are all open subsets. The algebras of tautological classes 20

$R^{*}\left(\mathcal{M}_{g, n}\right) \subset A^{*}\left(\mathcal{M}_{g, n}\right), R^{*}\left(\mathcal{M}_{g, n}^{\mathrm{rt}}\right) \subset A^{*}\left(\mathcal{M}_{g, n}^{\mathrm{rt}}\right), R^{*}\left(\mathcal{M}_{g, n}^{\mathrm{ct}}\right) \subset A^{*}\left(\mathcal{M}_{g, n}^{\mathrm{ct}}\right)$

are defined in each case as the image of the respective restriction of

$$
R^{*}\left(\overline{\mathcal{M}}_{g, n}\right) \subset A^{*}\left(\overline{\mathcal{M}}_{g, n}\right) .
$$

A basic question here concerns the extension of tautological relations over the boundary.

Q7. Does every tautological relation in $R^{*}\left(\mathcal{M}_{g, n}\right), R^{*}\left(\mathcal{M}_{g, n}^{\mathrm{rt}}\right)$, and $R^{*}\left(\mathcal{M}_{g, n}^{\text {ct }}\right)$ arise from the restriction of a tautological relation in $R^{*}\left(\overline{\mathcal{M}}_{g, n}\right)$ ?

If the answers to $\mathbf{Q 6}$ and $\mathbf{Q 7}$ are both affirmative, then the Pixton calculus determines the tautological rings in all the nonsingular and compact type cases (11).

The $n^{\text {th }}$ fiber product $\mathcal{C}_{g}^{n}$ of the universal curve

$$
\pi: \mathcal{C}_{g} \rightarrow \mathcal{M}_{g}
$$

is not an open set of $\overline{\mathcal{M}}_{g, n}$. However, there is a proper surjection

$$
\mathcal{M}_{g, n}^{\mathrm{rt}} \stackrel{\epsilon}{\longrightarrow} \mathcal{C}_{g}^{n}
$$

\footnotetext{
${ }^{20}$ See [9, 10, 37, 44, 84, 85] for various directions in the study of these tautological rings.
} 
The tautological ring $R^{*}\left(\mathcal{C}_{g}^{n}\right)$ is defined ${ }^{21}$ as the image of $R^{*}\left(\mathcal{M}_{g, n}^{\text {rt }}\right)$ under $\epsilon_{*}$. If the answers to $\mathbf{Q 6}$ and $\mathbf{Q 7}$ are both affirmative, then Pixton's calculus also determines $R^{*}\left(\mathcal{C}_{g}^{n}\right)$.

\subsection{Further directions.}

4.9.1. Symmetries. The symmetric group $\Sigma_{n}$ acts naturally on $\overline{\mathcal{M}}_{g, n}$ by permuting the $n$ markings. Since both $\mathcal{S}_{g, n}^{*}$ and $R^{*}\left(\overline{\mathcal{M}}_{g, n}\right)$ carry induced $\Sigma_{n}$-representations and

$$
q: \mathcal{S}_{g, n}^{*} \rightarrow R^{*}\left(\overline{\mathcal{M}}_{g, n}\right)
$$

is a morphism of $\Sigma_{n}$-representations, the ideal of tautological relations also carries an induced $\Sigma_{n}$-representation.

Pixton's relations interact in interesting ways with the $\Sigma_{n}$-action. For $g>0$, Pixton proves [79, Proposition 2] that the new relation ${ }^{22}$ in $\overline{\mathcal{P}}_{g, n}^{d}$ are generated by $\Sigma_{n}$-invariant tautological relations. For example, both Getzler's relation and the genus 2 relation displayed in Section 4.3 are new and invariant.

The symmetric group acts on the entire cohomology $H^{*}\left(\overline{\mathcal{M}}_{g, n}\right)$. For $g \leq 2$, the symmetric group representations are well understood [4, 23. 24, 27, 29, 76], and there is significant progress [5] in genus 3 . Constraints on the $\Sigma_{n}$-action on $R^{*}\left(\overline{\mathcal{M}}_{g, n}\right)$ are proven in [22, Section 4] and show certain cohomology classes can not be tautological.

4.9.2. Push-forward relations. A tautological relation in $\mathcal{S}_{g, n}^{*}$ yields a universal equation for the genus $\leq g$ Gromov-Witten theory of every target variety $X$ [51]. However, in genus 1, such tautological relations do not appear to be enough to prove the Virasoro constraints [17] for arbitrary targets [56]. Can any further universal equations in GromovWitten theory be found in the geometry of the moduli spaces of curves?

An idea to find further universal equations in Gromov-Witten theory using push-forwards is the following. Consider the gluing map,

$$
\delta: \overline{\mathcal{M}}_{g, 2} \rightarrow \overline{\mathcal{M}}_{g+1}
$$

\footnotetext{
${ }^{21}$ For another definition of $R^{*}\left(\mathcal{C}_{g}^{n}\right)$ and further study of the relationship with $R^{*}\left(\mathcal{M}_{g, n}^{\mathrm{rt}}\right)$, see [77].

${ }^{22}$ The new relations are those which do not lie in the $\mathbb{Q}$-span of relations coming from lower genus, lower marking number, or lower codimension.
} 
with image equal to the divisor $\Delta_{0} \subset \overline{\mathcal{M}}_{g}$ of curves with a nonseparating node. Elements of the kernel

$$
\delta_{*}: R^{*}\left(\overline{\mathcal{M}}_{g, 2}\right) \rightarrow R^{*+1}\left(\overline{\mathcal{M}}_{g+1}\right)
$$

yield universal equations for the genus $\leq g+1$ Gromov-Witten theory of targets $X$ with no genus $g+1$ terms. Hence, elements of $\operatorname{ker}\left(\delta_{*}\right)$ yields universal equations for genus $\leq g$ Gromov-Witten theory.

To formalize the notion of a push-forward relation, consider the composition

$$
\mathcal{S}_{g, 2}^{*} \stackrel{q}{\longrightarrow} R^{*}\left(\overline{\mathcal{M}}_{g, 2}\right) \stackrel{\delta_{*}}{\longrightarrow} R^{*+1}\left(\overline{\mathcal{M}}_{g+1}\right) .
$$

We have $\operatorname{ker}(q) \subset \operatorname{ker}\left(\delta_{*} \circ q\right)$. The gluing map $\delta$ lifts to

$$
\widetilde{\delta}: \mathcal{S}_{g, 2}^{*} \rightarrow \mathcal{S}_{g+1}^{*+1}
$$

and we also have $\operatorname{ker}(\widetilde{\delta}) \subset \operatorname{ker}\left(\delta_{*} \circ q\right)$. The subspace

$$
\operatorname{ker}(q)+\operatorname{ker}(\widetilde{\delta}) \subset \operatorname{ker}\left(\delta_{*} \circ q\right)
$$

yields relations in Gromov-Witten theory already captured before considering $\delta$.

A push-forward relation is a nonzero element of

$$
\frac{\operatorname{ker}\left(\delta_{*} \circ q\right)}{\operatorname{ker}(q)+\operatorname{ker}(\widetilde{\delta})} .
$$

Push-forward relations yield universal contraints in Gromov-Witten theory which appear to go beyond the contraints obtained from tautological relations in $\mathcal{S}_{g, n}^{*}$.

Are there any push-forward relations? Possible candidates were found in [57]. Let $r$ be even and satisfy $2 \leq r \leq g-1$. Define

$$
\chi_{g, r}=\sum_{a+b=2 g+r}(-1)^{a}\left[\Gamma_{\bullet}, \psi_{1}^{a} \psi_{2}^{b}\right] \in \mathcal{S}_{g, 2}^{2 g+r} .
$$

Here, $\Gamma \bullet$ is the unique graph with no edges. By [57, Theorem 2],

$$
\delta_{*} q\left(\chi_{g, r}\right)=0 .
$$

Moreover, the results of [57] show

$$
\chi_{g, r} \notin \operatorname{ker}(q) \text { and } \chi_{g, r} \notin \operatorname{ker}(\widetilde{\delta}),
$$

but whether $\chi_{g, r}$ ever avoids the sum $\operatorname{ker}(q)+\operatorname{ker}(\widetilde{\delta})$ is open. 
Pixton's relations can be used to search for push-forward relations (via several related constructions) which have the possibility of producing new universal Gromov-Witten equations. What role such relations will play in Gromov-Witten theory is not yet known.

\section{Double RAMificAtion CYCLES}

5.1. Overview. Curves of genus $g$ which admit a map to $\mathbb{P}^{1}$ with specified ramification profile $\mu$ over $0 \in \mathbb{P}^{1}$ and $\nu$ over $\infty \in \mathbb{P}^{1}$ define a double ramification cycle $\operatorname{DR}_{g}(\mu, \nu)$ on the moduli space of curves. The restriction of the double ramification cycle to the moduli space of nonsingular curves is a classical topic related to the linear equivalence of divisors.

The cycle $\mathrm{DR}_{g}(\mu, \nu)$ on the moduli space of stable curves is defined via the virtual fundamental class of the moduli space of stable maps to rubber. An explicit formula for $\operatorname{DR}_{g}(\mu, \nu)$ in the tautological ring, conjectured by Pixton [81] in 2014 and proven in [43] in 2015, is presented here. Pixton's double ramification formula expresses the cycle as a sum over stable graphs (corresponding to strata classes) with summands given by a product of leg and edge factors. The result shows how the calculus of tautological classes works in practice.

5.2. Moduli of relative stable maps. Let $\mu=\left(\mu_{1}, \ldots, \mu_{\ell(\mu)}\right)$ and $\nu=\left(\nu_{1}, \ldots, \nu_{\ell(\nu)}\right)$ be partitions of equal size,

$$
\sum_{i=1}^{\ell(\mu)} \mu_{i}=\sum_{j=1}^{\ell(\nu)} \nu_{j} .
$$

Let $C$ be a genus $g$ curve. We consider maps

$$
f: C \rightarrow \mathbb{P}^{1}
$$

with ramification profiles $\mu$ over $0 \in \mathbb{P}^{1}$ and and $\nu$ over $\infty \in \mathbb{P}^{1}$. Two such maps

$$
C \stackrel{f}{\rightarrow} \mathbb{P}^{1}, \quad C \stackrel{\widehat{f}}{\longrightarrow} \mathbb{P}^{1}
$$

are declared equivalent if $f$ and $\widehat{f}$ differ by a reparameterization of the target which keeps both 0 and $\infty \in \mathbb{P}^{1}$ fixed. 


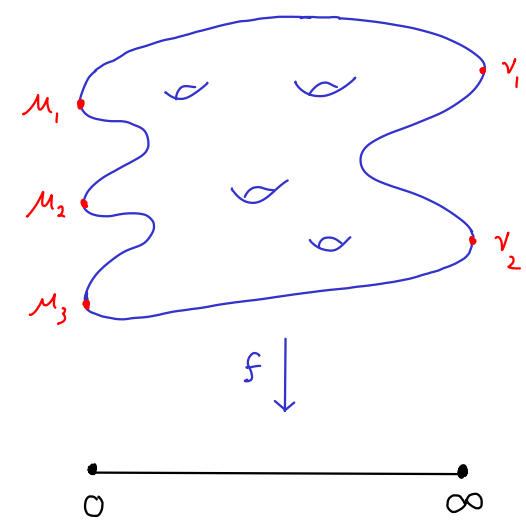

A natural compact moduli space of such maps $f$ arises in GromovWitten theory. Let

$$
\overline{\mathcal{M}}_{g}\left(\mathbb{P}^{1}, \mu, \nu\right)^{\sim}
$$

be the moduli space of stable relative maps to rubber with ramification profiles $\mu$ and $\nu$. In the moduli of relative stable maps, $f$ may degenerate in several ways: the domain $C$ may acquire nodes, $f$ may be constant on irreducible components of $C$, and the target $\mathbb{P}^{1}$ may degenerate. The first two phenomena are illustrated in the following diagram.

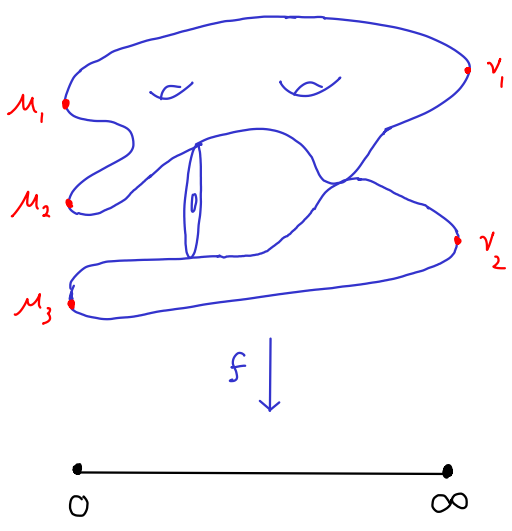

We refer the reader to [53, 54, 55] for the basic definitions of relative Gromov-Witten theory and the foundational development. 
5.3. Double ramification cycles. There is a natural morphism

$$
\rho: \overline{\mathcal{M}}_{g}\left(\mathbb{P}^{1}, \mu, \nu\right)^{\sim} \rightarrow \overline{\mathcal{M}}_{g, \ell(\mu)+\ell(\nu)}
$$

forgetting everything except the marked domain curve. The double ramification cycle is the push-forward of the virtual fundamental clas: ${ }^{23}$

$$
\mathrm{DR}_{g}(\mu, \nu)=\rho_{*}\left[\overline{\mathcal{M}}_{g}\left(\mathbb{P}^{1}, \mu, \nu\right)^{\sim}\right]^{v i r} \in A^{g}\left(\overline{\mathcal{M}}_{g, \ell(\mu)+\ell(\nu)}\right)
$$

Eliashberg asked in 2001: what is the formula for $\operatorname{DR}_{g}(\mu, \nu)$ ? As a first step, the double ramification cycle was proven to lie in the tautological ring in [21] in 2005,

$$
\operatorname{DR}_{g}(\mu, \nu) \in R^{g}\left(\overline{\mathcal{M}}_{g, \ell(\mu)+\ell(\nu)}\right) .
$$

The restriction of $\mathrm{DR}_{g}(\mu, \nu)$ to the moduli space $\mathcal{M}_{g, \ell(\mu)+\ell(\nu)}^{\mathrm{ct}}$ of curves of compact type can be calculated via the geometry of the universal Jacobian ${ }^{24}$ The result is Hain's formula [35, 36].

\footnotetext{
${ }^{23}$ The expected dimension of $\overline{\mathcal{M}}_{g}\left(\mathbb{P}^{1}, \mu, \nu\right) \sim$ is $3 g-3+\ell(\mu)+\ell(\nu)-g$ where $g$ is the dimension of the Jacobian of the domain curve.

${ }^{24}$ The matching on the moduli space of curves of compact type of the definitions of the double ramification cycle via the virtual class and the Jacobian geometry is not trivial and is proven in [11, 62.
} 


\subsection{Pixton's formula.}

5.4.1. Ramification vector. We place the ramification data in a vector

$$
\left(\mu_{1}, \ldots, \mu_{\ell(\mu)},-\nu_{1}, \ldots,-\nu_{\ell(\nu)}\right) \text {. }
$$

For any vector $S=\left(s_{1}, \ldots, s_{n}\right)$ with $\sum_{i} s_{i}=0$, we have

$$
\mathrm{DR}_{g, S} \in R^{g}\left(\overline{\mathcal{M}}_{g, n}\right) .
$$

The positive parts of $S$ specify ramification over $0 \in \mathbb{P}^{1}$ and the negative parts specify ramification over $\infty \in \mathbb{P}^{1}$. Free points corresponding to part of $S$ equal to 0 are also permitted.

Pixton [81] conjectured a beautiful formula for $\mathrm{DR}_{g, S} \in R^{g}\left(\overline{\mathcal{M}}_{g, n}\right)$ which involves a sum over admissible weightings of stable graphs.

5.4.2. Admissible weightings. Let $S=\left(s_{1}, \ldots, s_{n}\right)$ be double ramification data. Let $\Gamma \in \mathrm{G}_{g, n}$ be a stable graph of genus $g$ with $n$ legs. An admissible weighting is a function on the set of half-edges,

$$
w: \mathrm{H}(\Gamma) \rightarrow \mathbb{Z},
$$

which satisfies:

(i) $\forall h_{i} \in \mathrm{L}(\Gamma), w\left(h_{i}\right)=s_{i}$,

(ii) $\forall e \in \mathrm{E}(\Gamma)$ consisting of the half-edges $h(e), h^{\prime}(e) \in \mathrm{H}(\Gamma)$,

$$
w(h)+w\left(h^{\prime}\right)=0
$$

(iii) $\forall v \in \mathrm{V}(\Gamma), \sum_{v(h)=v} w(h)=0$.

A stable graph $\Gamma$, however, may have infinitely many admissible weightings $w$.

In order to regularize the sum over admissible weightings, Pixton introduced a regularization parameter

$$
r \in \mathbb{Z}_{>0} .
$$

An admissible weighting $\bmod r$ of $\Gamma$ is a function,

$$
w: \mathrm{H}(\Gamma) \rightarrow\{0, \ldots, r-1\},
$$

which satisfies 25 the conditions of (i-iii) above $\bmod r$. Let $\mathrm{W}_{\Gamma, r}$ be the set of admissible weightings $\bmod r$ of $\Gamma$. The set $\mathbf{W}_{\Gamma, r}$ is finite and can be summed over.

\footnotetext{
${ }^{25}$ For example, for (i), we require $w\left(h_{i}\right) \equiv s_{i} \bmod r$.
} 
5.4.3. Formula. Let $r$ be a positive integer. We denote by

$$
\mathrm{Q}_{g, S}^{d, r} \in R^{d}\left(\overline{\mathcal{M}}_{g, n}\right)
$$

the degree $d$ component of the class

$$
\begin{aligned}
\sum_{\Gamma \in \mathrm{G}_{g, n}} \sum_{w \in \mathrm{W}_{\Gamma, r}} \frac{1}{|\operatorname{Aut}(\Gamma)|} \frac{1}{r^{h^{1}(\Gamma)}} \xi_{\Gamma *}\left[\prod_{i=1}^{n} \exp \left(s_{i}{ }^{2} \psi_{h_{i}}\right) .\right. \\
\left.\prod_{e=\left(h, h^{\prime}\right) \in \mathrm{V}(\Gamma)} \frac{1-\exp \left(-w(h) w\left(h^{\prime}\right)\left(\psi_{h}+\psi_{h^{\prime}}\right)\right)}{\psi_{h}+\psi_{h^{\prime}}}\right] .
\end{aligned}
$$

For fixed $g, S$, and $d$, the class $\mathrm{Q}_{g, S}^{d, r} \in R^{d}\left(\overline{\mathcal{M}}_{g, n}\right)$ is polynomial in $r$ for sufficiently large $r$, see [43, Appendix]. We denote by $\mathrm{P}_{g, S}^{d}$ the value at $r=0$,

$$
\mathrm{P}_{g, S}^{d}=\left.\mathrm{Q}_{g, S}^{d, r}\right|_{r=0}
$$

Hence, $\mathrm{P}_{g, S}^{d}$ is the constant term.

Theorem 6 (Janda-P.-Pixton-Zvonkine 2015). For all $g \geq 0$ and double ramification data $S$,

$$
\mathrm{DR}_{g, S}=2^{-g} \mathrm{P}_{g, S}^{g} \in R^{g}\left(\overline{\mathcal{M}}_{g, n}\right)
$$

When restricted to $\mathcal{M}_{g, n}^{\mathrm{ct}}$, Theorem 6 recovers Hain's formula.

The proof of Theorem 6 uses the equivariant Gromov-Witten theory of $\mathbb{P}^{1}$ with:

- an orbifold $B \mathbb{Z}_{r}$-point at $0 \in \mathbb{P}^{1}$,

- a relative point at $\infty \in \mathbb{P}^{1}$.

Hence, orbifold GW theory [1, 12], relative GW theory [53, 54, 55], and the virtual localization formula [32] all play a role. Over $B \mathbb{Z}_{r}$, HurwitzHodge integrals arise exactly in the form of [45] and are analysed via Chiodo's formula [13]. The double ramification cycle arises over the relative point $\infty \in \mathbb{P}^{1}$.

In addition to conjecturing the formula of Theorem 6, Pixton [81] conjectured the following vanishing proven in [14].

Theorem 7 (Clader-Janda 2015). For all $g \geq 0$, double ramification data $S$, and $d>g$,

$$
\mathrm{P}_{g, S}^{d}=0 \in R^{d}\left(\overline{\mathcal{M}}_{g, n}\right)
$$


Clader and Janda further prove that the tautological relations obtained from Theorem 7 are a consequence of Pixton's set $\overline{\mathcal{P}}$ discussed in Section 4.6. For $d<g$, the classes $\mathrm{P}_{g, S}^{d}$ do not yet have a geometric interpretation.

5.5. The Hodge bundle. Let $g \geq 2$ and $S=\emptyset$, so $\mu=\nu=\emptyset$. Then, the morphism $\rho$ from the moduli of stable maps to the moduli of curves is an isomorphism,

$$
\rho: \overline{\mathcal{M}}_{g}\left(\mathbb{P}^{1}, \emptyset, \emptyset\right)^{\sim} \stackrel{\sim}{\longrightarrow} \overline{\mathcal{M}}_{g} .
$$

By a study of the obstruction theory,

$$
\mathrm{DR}_{g, \emptyset}=(-1)^{g} \lambda_{g} \in R^{g}\left(\overline{\mathcal{M}}_{g}\right),
$$

where $\lambda_{g}$ is the top Chern class of the Hodge bundle

$$
\mathbb{E} \rightarrow \overline{\mathcal{M}}_{g}
$$

Pixton's formula in the $S=\emptyset$ case therefore yields an expression for $\lambda_{g}$. By the analysis of [43, Section 0.5.3], the result is a new and very special formula for $\lambda_{g}$ : all the strata which appear are supported on

$$
\Delta_{0} \subset \overline{\mathcal{M}}_{g}
$$

the divisor with a nonseparating node.26

In the diagram ${ }^{27}$ below, each labeled graph $\Gamma$ describes a moduli space $\overline{\mathcal{M}}_{\Gamma}$, a tautological class $\gamma$, and a natural map

$$
\xi_{\Gamma}: \overline{\mathcal{M}}_{\Gamma} \rightarrow \overline{\mathcal{M}}_{g}
$$

The convention in the diagrams is that the labeled graph represents the cycle class

$$
\xi_{\Gamma *}(\gamma)=q([\Gamma, \gamma])
$$

see Section 4.3.

\footnotetext{
${ }^{26}$ In particular, the vanishing

$$
\lambda_{g}^{2}=0 \in R^{*}\left(\overline{\mathcal{M}}_{g}\right)
$$

is an immediate consequence since $\left.\lambda_{g}\right|_{\Delta_{0}}=0$.

${ }^{27}$ The diagrams (taken from [4]) are computed using code written by A. Pixton. The artistic display is due to F. Janda.
} 
The formulas for $\lambda_{2} \in R^{2}\left(\overline{\mathcal{M}}_{2}\right)$ and $\lambda_{3} \in R^{3}\left(\overline{\mathcal{M}}_{3}\right)$ are:

$$
\begin{aligned}
& \lambda_{2}=\frac{1}{240} \bigcirc+\frac{1}{1152} \bigcirc \\
& \lambda_{3}=\frac{1}{2016} \overbrace{2}^{2}+\frac{1}{2016} \overparen{\psi} \underset{\mathbf{3}}{\psi}-\frac{1}{672} \mathbf{0}^{\psi}+\frac{1}{5760} \\
& -\frac{13}{30240} \text { 0 }-\frac{1}{5760} \bigcirc+\frac{1}{82944} \bigcirc \text {. }
\end{aligned}
$$

More interesting is the formula for $\lambda_{4} \in R^{4}\left(\overline{\mathcal{M}}_{4}\right)$ :

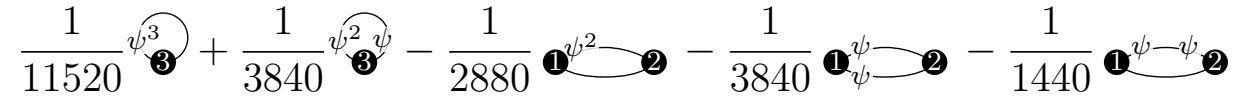

$$
\begin{aligned}
& -\frac{1}{1920} \mathbf{0}_{\psi}^{\psi}-\frac{1}{2880} \psi_{2}^{2}-\frac{1}{3840} \mathbf{Q}_{\mathbf{2}}^{2}+\frac{1}{48384} \psi_{2}^{2}
\end{aligned}
$$

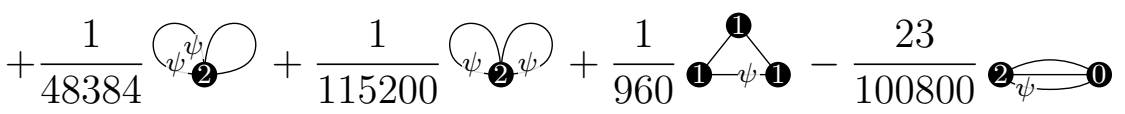

$$
\begin{aligned}
& -\frac{1}{57600} 2 \widehat{0}_{\psi}-\frac{1}{16128} 2-\frac{1}{16128} \mathbf{2}-\mathbf{0} \\
& -\frac{1}{57600} \bigcirc-\frac{1}{16128} \bigcirc-\frac{1}{16128} 0_{\psi} \bigcirc \\
& +\frac{23}{100800} \overbrace{2}^{0}+\frac{23}{50400}+\frac{1}{16128} \\
& +\frac{1}{276480} \bigcirc \psi-\frac{13}{725760} \bigcirc \longrightarrow \frac{1}{138240} \bigcirc \\
& -\frac{43}{1612800} \rightleftharpoons \mathbf{0}-\frac{13}{725760} \bigcirc \mathbf{0} \\
& -\frac{1}{276480} \bigcirc-\frac{1}{7962624} \bigcirc
\end{aligned}
$$


5.6. Further formulas. Pixton's formula for the double ramification cycle was presented here as an example. Several other formulas have been recently studied (the Chern character of the Verlinde bundle [61, the cycle class of the loci of holomorphic/meromorphic differentials [25. Appendix]). The form of a summation over stable graphs $G_{g, n}$ with summands given by a product over vertex, leg, and edge factors is ubiquitous (and reminiscent of Feynman expansions of integrals in quantum field theory).

\section{REFERENCES}

[1] D. Abramovich, T. Graber, and A. Vistoli, Gromov-Witten theory for Deligne-Mumford stacks, Amer. J. Math. 130 (2008), 1337-1398.

[2] K. Behrend, Gromov-Witten invariants in algebraic geometry, Invent. Math. 127 (1997), 601-617.

[3] K. Behrend and B. Fantechi, The intrinsic normal cone, Invent. Math. 128 (1997), 45-88.

[4] J. Bergström, C. Faber, and G. van der Geer, Siegel modular forms of genus 2 and level 2: cohomological computations and conjectures, IMRN 100 (2008).

[5] J. Bergström, C. Faber, and G. van der Geer, Siegel modular forms of degree 3 and the cohomology of local systems, Selecta Mathematica, 20 (2014), 83-124.

[6] P. Belorousski and R. Pandharipande, A descendent relation in genus 2, Ann. Scuola Norm. Sup. Pisa Cl. Sci. 29 (2000), 171-191.

[7] S. Boldsen, Improved homological stability for the mapping class group with integral or twisted coefficients, Math. Z. 270 (2012), 297-329.

[8] A. Buryak, F. Janda, and R. Pandharipande, The hypergeometric functions of the Faber-Zagier and Pixton relations, arXiv:1502.05150.

[9] A. Buryak, S. Shadrin, and D. Zvonkine, Top tautological group of $\mathcal{M}_{g, n}$, arXiv:1312.2775.

[10] R. Cavalieri and S. Yang, Tautological pairings on moduli spaces of curves, Proc. Amer. Math. Soc. 139 (2011), 51-62.

[11] R. Cavalieri, S. Marcus, and J. Wise, Polynomial families of tautological classes on $\mathcal{M}_{g, n}^{\mathrm{rt}}$, J. Pure and Applied Algebra 216 (2012), 950-981.

[12] W. Chen and Y. Ruan, Orbifold Gromov-Witten theory in Orbifolds in mathematics and physics (Madison, WI, 2001), 25-85, Contemp. Math. 310 (2002).

[13] A. Chiodo, Stable twisted curves and their r-spin structures, Ann. Inst. Fourier 58 (2008), 1635-1689.

[14] E. Clader and F. Janda, Pixton's double ramification cycle relations, arXiv:1601.02871.

[15] E. Eftekhary and I. Setayesh, On the structure of the $\kappa$ ring, arXiv:1207.2380.

[16] E. Eftekhary and I. Setayesh, On the $\kappa$ ring of $\overline{\mathcal{M}}_{g, n}$, arXiv:1307.2754. 
[17] T. Eguchi, K. Hori, and C.-S. Xiong, Quantum cohomology and Virasoro algebra, Phys. Lett. B402 (1997), 71-80.

[18] C. Faber, A conjectural description of the tautological ring of the moduli space of curves, Moduli of curves and abelian varieties, 109-129, Aspects Math., Vieweg, Braunschweig, 1999.

[19] C. Faber and R. Pandharipande (with an appendix by D. Zagier), Logarithmic series and Hodge integrals in the tautological ring, Michigan Math. J. 48 (2000), 215-252.

[20] C. Faber and R. Pandharipande, Hodge integrals, partition matrices, and the $\lambda_{g}$ conjecture, Ann. of Math. 157 (2003), 97-124.

[21] C. Faber and R. Pandharipande, Relative maps and tautological classes, JEMS 7 (2005), 13-49.

[22] C. Faber and R. Pandharipande, Tautological and non-tautological cohomology of the moduli space of curves in Handbook of moduli. Vol. I, 293-330, Adv. Lect. Math. 24, Int. Press: Somerville, MA, 2013.

[23] C. Faber and G. van der Geer, Sur la cohomologie des systèmes locaux sur les espaces des modules des courbes de genre 2 et des surfaces abéliennes I C. R. Acad. Sci. Paris, 338, 381-384.

[24] C. Faber and G. van der Geer, Sur la cohomologie des systèmes locaux sur les espaces des modules des courbes de genre 2 et des surfaces abéliennes II C. R. Acad. Sci. Paris, 338, 467-470.

[25] G. Farkas and R. Pandharipande, The moduli space of twisted canonical divisors, J. Institute Math. Jussieu (to appear).

[26] W. Fulton, Intersection theory, Springer-Verlag: Berlin, 1984.

[27] E. Getzler, Operads and moduli spaces of genus 0 Riemann surfaces in The moduli space of curves, 199-230, R. Dijkgraaf, C. Faber, and G. van der Geer, eds., Birkhäuser: Basel, 1995.

[28] E. Getzler, Intersection theory on $\overline{\mathcal{M}}_{1,4}$ and elliptic Gromov-Witten invariants, J. Amer. Math. Soc. 10 (1997), 973-998.

[29] E. Getzler, The semi-classical approximation for modular operads, Comm. Math. Phys. 194 (1998), 481-492.

[30] E. Getzler, Topological recursion relations in genus 2 in Integrable systems and algebraic geometry (Kobe/Kyoto 1997), 73-106, World Scientific Publishing: River Edge, NJ, 1998.

[31] E. Getzler and R. Pandharipande, Virasoro constraints and the Chern classes of the Hodge bundle, Nuclear Phys. B 530 (1998), 701-714.

[32] T. Graber and R. Pandharipande, Localization of virtual classes, Invent. Math. 135 (1999), 487-518.

[33] T. Graber and R. Pandharipande, Constructions of nontautological classes on moduli spaces of curves, Michigan Math. J. 51 (2003), 93-109.

[34] T. Graber and R. Vakil, Relative virtual localization and vanishing of tautological classes on moduli spaces of curves, Duke Math. J. 130 (2005), $1-37$.

[35] S. Grushevsky and D. Zakharov, The double ramification cycle and the theta divisor, Proc. Amer. Math. Soc. 142 (2014), 4053-4064.

[36] R. Hain, Normal functions and the geometry of moduli spaces of curves in Handbook of moduli. Vol. I, 527-578, Adv. Lect. Math. 24, Int. Press: Somerville, MA, 2013. 
[37] E. Ionel, Topological recursive relations in $H^{2 g}\left(\mathcal{M}_{g, n}\right)$, Invent. Math. 148 (2002), 627-658.

[38] E. Ionel, Relations in the tautological ring of $\mathcal{M}_{g}$, Duke Math. J. 129 (2005), 157-186.

[39] F. Janda, Tautological relations in moduli spaces of weighted pointed curves, arXiv:1306.6580.

[40] F. Janda, Comparing tautological relations from the equivariant GromovWitten theory of projective spaces and spin structures, arXiv:1407.4778.

[41] F. Janda, Relations in the tautological rings and Frobenius manifolds near the discriminant, arXiv:1505.03419.

[42] F. Janda, Relations on $\overline{\mathcal{M}}_{g, n}$ via equivariant Gromov-Witten theory of $\mathbb{P}^{1}$, arXiv:1509.08421

[43] F. Janda, R. Pandharipande, A. Pixton, and D. Zvonkine, Double ramification cycles on moduli spaces of curves, arXiv:1602.04705.

[44] F. Janda and A. Pixton, Socle pairings on tautological rings, arXiv:1304.0026.

[45] P. Johnson, R. Pandharipande, and H.-H. Tseng, Abelian Hurwitz-Hodge integrals, Michigan Math. J. 60 (2011), 171-198.

[46] M. E. Kazarian and S. K. Lando, An algebro-geometric proof of Witten's conjecture, J. Amer. Math. Soc. 20 (2007), 1079-1089.

[47] S. Keel, Intersection theory of moduli space of stable n-pointed curves of genus zero, Trans. Amer. Math. Soc. 330 (1992), 545-574.

[48] T. Kimura and X. Liu, A genus 3 topological recursion relation, Comm. Math. Phys. 262 (2006), 645-661.

[49] T. Kimura and X. Liu, Topological recursion relations on $\overline{\mathcal{M}}_{3,2}$, Science China Mathematics 58 (2015), 1909-1922.

[50] M. Kontsevich, Intersection theory on the moduli space of curves and the matrix Airy function, Comm. Math. Phys. 147 (1992), 1-23.

[51] M. Kontsevich and Yu. Manin, Gromov-Witten classes, quantum cohomology, and enumerative geometry, Comm. Math. Phys. 164 (1994), 525-562.

[52] M. Kontsevich and Yu. Manin, Quantum cohomology of a product with an appendix by R. Kaufmann, Invent. Math. 124 (1996), 313-339.

[53] A.-M. Li and Y. Ruan, Symplectic surgery and Gromov-Witten invariants of Calabi-Yau 3-folds, Invent. Math. 145 (2001), 151-218.

[54] J. Li, Stable morphisms to singular schemes and relative stable morphisms, J. Differential Geom. 57 (2001), 509-578.

[55] J. Li, Lecture notes on relative $G W$-invariants, http://users.ictp.it/ pub_off/lectures/lns019/Jun_Li/Jun_Li.pdf

[56] X. Liu, Elliptic Gromov-Witten invariants and Virasoro conjecture, Comm. Math. Phys. 216 (2001), 705-728.

[57] X. Liu and R. Pandharipande, New topological recursion relations, J. Algebraic Geom. 20 (2011), 479-494.

[58] E. Looijenga, On the tautological ring of $M_{g}$. Invent. Math. 121 (1995), 411-419.

[59] I. Madsen and M. Weiss, The stable moduli space of Riemann surfaces: Mumford's conjecture, Ann. of Math. 165 (2007), 843-941.

[60] A. Marian, D. Oprea, and R. Pandharipande, The moduli space of stable quotients, Geom. Topol. 15 (2011), 1651-1706. 
[61] A. Marian, D. Oprea, R. Pandharipande, A. Pixton, and D. Zvonkine, The Chern character of the Verlinde bundle over the moduli space of curves, J. Reine Angew. Math. (to appear).

[62] S. Marcus and J. Wise, Stable maps to rational curves and the relative Jacobian, arXiv:1310.5981.

[63] M. Mirzakhani, Weil-Petersson volumes and intersection theory on the moduli space of curves, J. Amer. Math. Soc. 20 (2007), 1-23.

[64] S. Morita, Generators for the tautological algebra of the moduli space of curves, Topology 42 (2003), 787-819.

[65] D. Mumford, Towards an enumerative geometry of the moduli space of curves in Arithmetic and Geometry, 271-328, M. Artin and J. Tate, eds., Part II, Birkhäuser: Berlin, 1983.

[66] A. Okounkov and R. Pandharipande, Gromov-Witten theory, Hurwitz numbers, and matrix models. Algebraic geometry, Seattle 2005. Proc. Sympos. Pure Math., 80, Part 1, 325-414.

[67] R. Pandharipande, A geometric construction of Getzler's elliptic relation, Math. Ann. 313 (1999), 715-729.

[68] R. Pandharipande, The $\kappa$ classes on the moduli spaces of curves, arXiv:1108.5984.

[69] $\mathrm{R}$. Pandharipande, The $\kappa$ ring of the moduli of curves of compact type, Acta Math. 208 (2012), 335-388.

[70] R. Pandharipande and A. Pixton, Relations in the tautological ring, arXiv:1101.2236.

[71] R. Pandharipande and A. Pixton, Relations in the tautological ring of the moduli space of curves, arXiv:1301.4561.

[72] R. Pandharipande, A. Pixton, and D. Zvonkine, Relations on $\overline{\mathcal{M}}_{g, n}$ via 3-spin structures, J. Amer. Math. Soc. 28 (2015), 279-309.

[73] R. Pandharipande, A. Pixton, and D. Zvonkine, Tautological relations via $r$-spin structures, in preparation.

[74] D. Petersen, The tautological ring of the space of pointed genus two curves of compact type, arXiv:1310.7369.

[75] D. Petersen, The structure of the tautological ring in genus one, Duke Math. J. 163 (2014), 777-793.

[76] D. Petersen, Cohomology of local systems on the moduli of principally polarized abelian surfaces, Pacific J. Math. 275 (2015), 39-61.

[77] D. Petersen, Poincaré duality of wonderful compactifications and tautological rings, IMRN 296 (2015).

[78] D. Petersen and O. Tommasi, The Gorenstein conjecture fails for the tautological ring of $\overline{\mathcal{M}}_{2, n}$, Invent. Math. 196 (2014), 139-161.

[79] A. Pixton, Conjectural relations in the tautological ring of $\overline{\mathcal{M}}_{g, n}$, arXiv:1207.1918.

[80] A. Pixton, The tautological ring of the moduli space of curves, Princeton Ph.D 2013.

[81] A. Pixton, Double ramification cycles and tautological relations on $\overline{\mathcal{M}}_{g, n}$, unpublished notes 2014.

[82] O. Randal-Williams, Relations among tautological classes revisited, Adv. Math. 231 (2012), 1773-1785. 
[83] I. Setayesh, The product structure of the $\kappa$ ring of the moduli of curves of compact type, Einstein seminar, Humboldt Univ. Berlin (Feb 11, 2016).

[84] M. Tavakol, The tautological ring of $\mathcal{M}_{1, n}^{\text {ct }}$, Ann. Inst. Fourier (Grenoble) 61 (2011), 2751-2779.

[85] M. Tavakol, The tautological ring of the moduli space $\mathcal{M}_{2, n}^{\mathrm{rt}}$, IMRN 24 (2014), 6661-6683.

[86] C. Teleman, The structure of $2 D$ semi-simple field theories, Invent. Math. 188 (2012), 525-588.

[87] Q. Yin, Cycles on curves and Jacobians: a tale of two tautological rings, arXiv:1407.2216.

[88] E. Witten, Two dimensional gravity and intersection theory on moduli space, Surveys in Diff. Geom. 1 (1991), 243-310.

[89] S. Zhu, A note on the relations in the tautological ring of $\overline{\mathcal{M}}_{g}$, Pacific J. Math. 252 (2011), 499-510.

Departement Mathematik

ETH Zürich

Rämistrasse 101

8092 Zürich, Switzerland 